\title{
LOGARITHMIC DECAY OF THE ENERGY FOR AN HYPERBOLIC-PARABOLIC COUPLED SYSTEM
}

\author{
InES KAMOUN FATHALLAH ${ }^{1}$
}

\begin{abstract}
This paper is devoted to the study of a coupled system which consists of a wave equation and a heat equation coupled through a transmission condition along a steady interface. This system is a linearized model for fluid-structure interaction introduced by Rauch, Zhang and Zuazua for a simple transmission condition and by Zhang and Zuazua for a natural transmission condition. Using an abstract theorem of Burq and a new Carleman estimate proved near the interface, we complete the results obtained by Zhang and Zuazua and by Duyckaerts. We prove, without a Geometric Control Condition, a logarithmic decay of the energy.
\end{abstract}

Mathematics Subject Classification. 37L15, 35B37, 74F10, 93D20.

Received December 24, 2008. Revised November 14, 2009 and March 31, 2010.

Published online August 6, 2010.

\section{INTRODUCTION AND RESULTS}

In this work, we are interested in a linearized model for fluid-structure interaction introduced by Zhang and Zuazua in [15] and Duyckaerts in [6]. This model consists of a wave equation and a heat equation coupled through an interface by suitable transmission conditions. Our purpose is to analyze the stability of this system and therefore to determine the decay rate of the energy of solutions as $t \rightarrow \infty$.

Let $\Omega \subset \mathbb{R}^{n}$ be a bounded domain with a smooth boundary $\Gamma=\partial \Omega$. Let $\Omega_{1}$ and $\Omega_{2}$ be two bounded open sets with smooth boundary such that $\Omega_{1} \subset \Omega$ and $\Omega_{2}=\Omega \backslash \bar{\Omega}_{1}$. We denote by $\gamma=\partial \Omega_{1} \cap \partial \Omega_{2}$ the interface, $\gamma \subset \subset \Omega, \Gamma_{j}=\partial \Omega_{j} \backslash \gamma, j=1,2$, and we suppose that $\Gamma_{2} \neq \emptyset$. Let $\partial_{n}$ and $\partial_{n^{\prime}}$ the unit outward normal vectors of $\Omega_{1}$ and $\Omega_{2}$ respectively. We recall that $\partial_{n^{\prime}}=-\partial_{n}$ on $\gamma$.

$$
\begin{cases}\partial_{t} u-\triangle u=0 & \text { in }(0, \infty) \times \Omega_{1}, \\ \partial_{t}^{2} v-\Delta v=0 & \text { in }(0, \infty) \times \Omega_{2}, \\ u=0 & \text { on }(0, \infty) \times \Gamma_{1}, \\ v=0 & \text { on }(0, \infty) \times \Gamma_{2}, \\ u=\partial_{t} v, \quad \partial_{n} u=-\partial_{n^{\prime}} v & \text { on }(0, \infty) \times \gamma, \\ \left.u\right|_{t=0}=u_{0} \in L^{2}\left(\Omega_{1}\right) & \text { in } \Omega_{1}, \\ \left.v\right|_{t=0}=v_{0} \in H^{1}\left(\Omega_{2}\right),\left.\quad \partial_{t} v\right|_{t=0}=v_{1} \in L^{2}\left(\Omega_{2}\right) & \text { in } \Omega_{2} .\end{cases}
$$

Keywords and phrases. Fluid-structure interaction, wave-heat model, stability, logarithmic decay.

1 Laboratoire LMV, Université de Versailles Saint-Quentin-en-Yvelines, 45 Avenue des États-Unis, Bâtiment Fermat, 78035 Versailles, France. ines.fathallah@math.uvsq.fr 
In this system, $u$ may be viewed as the velocity of fluid; while $v$ and $\partial_{t} v$ represent respectively the displacement and velocity of the structure. That's why the transmission condition $u=\partial_{t} v$ is considered as the natural condition. For the discussion of this model, we refer to [12,15].

System (1.1) is introduced by Zhang and Zuazua [15]. The same system is considered by Rauch et al. in [12] but for the simplified transmission condition $u=v$ on the interface instead of $u=\partial_{t} v$. They prove, under a suitable Geometric Control Condition (GCC) (see [1]), a polynomial decay result. Zhang and Zuazua in [15] prove, without the GCC, a logarithmic decay result. Duyckaerts in [6] improves these results.

For system (1.1), Zhang and Zuazua in [15], prove the lack of uniform decay and, under the GCC, a polynomial decay result. Without geometric conditions, they analyze the difficulty to prove the logarithmic decay result. This difficulty is mainly due to the lack of regularity gain of the wave component $v$ near the interface $\gamma$ (see [15], Rem. 19) which means that the embedding of the domain $D(\mathcal{A})$ of the dissipative operator in the energy space is not compact (see [15], Thm. 1). In [6], Duyckaerts improves the polynomial decay result under the GCC and confirms the same obstacle to proving the logarithmic decay for solution of (1.1) without the GCC. In this paper we are interested in this problem.

There is an extensive literature on the stabilization of PDEs and on the Logarithmic decay of the energy $([2-4,7,9,11,13]$ and the references cited therein) and this paper uses part of the idea developed in [3].

Here we recall the mathematical framework for this problem (see [15]).

Define the energy space $H$ and the operator $\mathcal{A}$ on $H$ with domain $D(\mathcal{A})$ by

$$
H=\left\{F=\left(f_{1}, f_{2}, f_{3}\right) \in L^{2}\left(\Omega_{1}\right) \times H_{\Gamma_{2}}^{1}\left(\Omega_{2}\right) \times L^{2}\left(\Omega_{2}\right)\right\}
$$

where $H_{\Gamma_{2}}^{1}\left(\Omega_{2}\right)$ is defined as the space

$$
\begin{aligned}
H_{\Gamma_{2}}^{1}\left(\Omega_{2}\right) & =\left\{f \in H^{1}\left(\Omega_{2}\right),\left.f\right|_{\Gamma_{2}}=0\right\}, \\
\mathcal{A} F & =\left(\triangle f_{1}, f_{3}, \triangle f_{2}\right) \\
D(\mathcal{A}) & =\left\{F \in H, f_{1} \in H^{1}\left(\Omega_{1}\right), \triangle f_{1} \in L^{2}\left(\Omega_{1}\right),\right. \\
f_{3} \in H_{\Gamma_{2}}^{1}\left(\Omega_{2}\right), \triangle f_{2} \in L^{2}\left(\Omega_{2}\right),\left.f_{1}\right|_{\gamma} & \left.=\left.f_{3}\right|_{\gamma},\left.\partial_{n} f_{1}\right|_{\gamma}=-\left.\partial_{n} f_{2}\right|_{\gamma}\right\} .
\end{aligned}
$$

Thus system (1.1) may be rewritten as an abstract Cauchy problem in $H$ as

$$
\left\{\begin{array}{l}
\partial_{t} U(t)=\mathcal{A} U(t), \quad t>0, \\
U(0)=U_{0}
\end{array}\right.
$$

where $U(t)=\left(u(t), v(t), \partial_{t} v(t)\right)$ and $U_{0}=\left(u_{0}, v_{0}, v_{1}\right)$.

The operator $\mathcal{A}$ is the generator of a strongly continuous semi-group (see [15], Thm. 1).

In our case, i.e. when $\Gamma_{2} \neq \emptyset$, the energy of any solution $U=\left(u, v, \partial_{t} v\right)$ of (1.2) is defined as one half of the square of a norm on $H$ and we have

$$
E(U(t))=\frac{1}{2}\left(\int_{\Omega_{1}}|u(t)|^{2} \mathrm{~d} x+\int_{\Omega_{2}}\left|\partial_{t} v(t)\right|^{2} \mathrm{~d} x+\int_{\Omega_{2}}|\nabla v(t)|^{2} \mathrm{~d} x\right) .
$$

When $\Gamma_{2}=\emptyset$, we refer to $[6,15]$.

By means of the classical energy method, we have

$$
\frac{\mathrm{d}}{\mathrm{d} t} E(U(t))=-\int_{\Omega_{1}}|\nabla u|^{2} \mathrm{~d} x
$$

Therefore the energy of (1.2) is decreasing with respect to $t$, the dissipation coming from the heat component $u$. Our main goal is to prove a logarithmic decay without the GCC assumption. 
As Duyckaerts [6] did for the simplified model, the idea is, first, to use a known result of Burq (see [5]) which links, for dissipative operators, logarithmic decay to resolvent estimates with exponential loss; secondly to prove, following the work of Bellassoued in [3], a new Carleman inequality near the interface $\gamma$.

The main results are the following Theorem 1.1 concerning the resolvent and Theorem 1.2 concerning the decay.

Theorem 1.1. There exists $C>0$, such that for every $\mu \in \mathbb{R}$, we have

$$
\left\|(\mathcal{A}-\mathrm{i} \mu)^{-1}\right\|_{\mathcal{L}(H)} \leq C \mathrm{e}^{C|\mu|} .
$$

Theorem 1.2. There exists $C>0$, such that for all $U_{0} \in D(\mathcal{A})$, we have

$$
\sqrt{E(U(t))} \leq \frac{C}{\log (t+2)}\left\|U_{0}\right\|_{D(\mathcal{A})}
$$

Remark 1.1. To simplify, we assumed that $\Gamma_{2} \neq \emptyset$. When $\Gamma_{2}$ is empty, the constant functions $(0, c, 0)$, where $c$ is arbitrary, are solutions of system (1.2). Therefore it is necessary to consider the decay of solutions orthogonal to $(0, c, 0)$ in $H$ (for more details we refer to Thm. 1 in [15]).

Burq in [5], Theorem 3, and Duyckaerts in [6], Section 7, show that to prove Theorem 1.2 it suffices to prove Theorem 1.1.

The strategy of the proof of Theorem 1.1, when $\mu \neq 0$, is the following. A new Carleman estimate proved near the interface $\gamma$ implies an interpolation inequality given by Theorem 2.2. Theorem 2.2 implies Theorem 2.1 which gives an estimate of the wave component by the heat one and which is the key point of the proof of Theorem 1.1.

The rest of this paper is organized as follows. In Section 2, we prove Theorem 1.1, for $\mu \neq 0$, from Theorem 2.1 and we explain how Theorem 2.2 implies Theorem 2.1. For $\mu=0$, the proof of Theorem 1.1 is given in Appendix C. In Section 3, we begin by stating the new Carleman estimate and we explain how this estimate implies Theorem 2.2. Then we give the proof of this Carleman estimate. Section 4 is devoted to the proof of important estimates, stated in Theorem 3.2, in the proof of this Carleman estimate. Appendices A and B are devoted to prove some technical results used along the paper.

\section{Proof of Theorem 1.1}

For $\mu=0$, the proof of Theorem 1.1 is given in Appendix C. For $\mu \neq 0$, we start by stating Theorem 2.1. Then we will explain how this theorem implies Theorem 1.1. Finally, we give the proof of Theorem 2.1.

Let $\mu_{0}>0$, small enough, for any $\mu$ such that $|\mu| \geq \mu_{0}$, we assume

$$
F=(\mathcal{A}-\mathrm{i} \mu) U, \quad U=\left(u_{0}, v_{0}, v_{1}\right) \in D(\mathcal{A}), \quad F=\left(f_{0}, g_{0}, g_{1}\right) \in H
$$

Equation (2.1) yields

$$
\left\{\begin{aligned}
(\triangle-\mathrm{i} \mu) u_{0} & =f_{0} & & \text { in } \Omega_{1}, \\
\left(\triangle+\mu^{2}\right) v_{0} & =g_{1}+\mathrm{i} \mu g_{0} & & \text { in } \Omega_{2}, \\
v_{1} & =g_{0}+\mathrm{i} \mu v_{0} & & \text { in } \Omega_{2},
\end{aligned}\right.
$$

with the following boundary conditions

$$
\left\{\begin{aligned}
\left.u_{0}\right|_{\Gamma_{1}} & =0,\left.\quad v_{0}\right|_{\Gamma_{2}}=0 \\
u_{0}-\mathrm{i} \mu v_{0} & =\left.g_{0}\right|_{\gamma}, \\
\partial_{n} u_{0}-\partial_{n} v_{0} & =\left.0\right|_{\gamma} .
\end{aligned}\right.
$$


Theorem 2.1. Let $U=\left(u_{0}, v_{0}, v_{1}\right) \in D(\mathcal{A})$ satisfy equations (2.2) and (2.3). Then there exist constants $C>0$, $c_{1}>0$ and $\mu_{0}>0$ such that for any $|\mu| \geq \mu_{0}$ we have the following estimate

$$
\left\|v_{0}\right\|_{H^{1}\left(\Omega_{2}\right)}^{2} \leq C \mathrm{e}^{c_{1}|\mu|}\left(\left\|f_{0}\right\|_{L^{2}\left(\Omega_{1}\right)}^{2}+\left\|g_{1}+\mathrm{i} \mu g_{0}\right\|_{L^{2}\left(\Omega_{2}\right)}^{2}+\left\|g_{0}\right\|_{H^{1}\left(\Omega_{2}\right)}^{2}+\left\|u_{0}\right\|_{H^{1}\left(\Omega_{1}\right)}^{2}\right) .
$$

Moreover, from the first equation of system (2.2), we have

$$
\int_{\Omega_{1}}(-\triangle+\mathrm{i} \mu) u_{0} \overline{u_{0}} \mathrm{~d} x=\left\|\nabla u_{0}\right\|_{L^{2}\left(\Omega_{1}\right)}^{2}+\mathrm{i} \mu\left\|u_{0}\right\|_{L^{2}\left(\Omega_{1}\right)}^{2}-\int_{\gamma} \partial_{n} u_{0} \overline{u_{0}} \mathrm{~d} \sigma .
$$

Since $\left.u_{0}\right|_{\gamma}=g_{0}+\mathrm{i} \mu v_{0}$ and $\partial_{n} u_{0}=-\partial_{n^{\prime}} v_{0}$, then

$$
\int_{\Omega_{1}}(-\triangle+\mathrm{i} \mu) u_{0} \bar{u}_{0} \mathrm{~d} x=\left\|\nabla u_{0}\right\|_{L^{2}\left(\Omega_{1}\right)}^{2}+\mathrm{i} \mu\left\|u_{0}\right\|_{L^{2}\left(\Omega_{1}\right)}^{2}-\mathrm{i} \mu \int_{\gamma} \partial_{n^{\prime}} v_{0} \bar{v}_{0} \mathrm{~d} \sigma+\int_{\gamma} \partial_{n^{\prime}} v_{0} \bar{g}_{0} \mathrm{~d} \sigma .
$$

From the second equation of system (2.2) and multiplying by $(-\mathrm{i} \mu)$, we obtain

$$
\mathrm{i} \mu \int_{\Omega_{2}}\left(\triangle+\mu^{2}\right) v_{0} \bar{v}_{0} \mathrm{~d} x=-\mathrm{i} \mu\left\|\nabla v_{0}\right\|_{L^{2}\left(\Omega_{2}\right)}^{2}+\mathrm{i} \mu^{3}\left\|v_{0}\right\|_{L^{2}\left(\Omega_{2}\right)}^{2}+\mathrm{i} \mu \int_{\gamma} \partial_{n^{\prime}} v_{0} \bar{v}_{0} \mathrm{~d} \sigma
$$

Adding (2.5) and (2.6), we obtain

$$
\begin{aligned}
\int_{\Omega_{1}}(-\triangle+\mathrm{i} \mu) u_{0} \bar{u}_{0} \mathrm{~d} x+\mathrm{i} \mu & \int_{\Omega_{2}}\left(\Delta+\mu^{2}\right) v_{0} \bar{v}_{0} \mathrm{~d} x \\
& =\mathrm{i} \mu\left\|u_{0}\right\|_{L^{2}\left(\Omega_{1}\right)}^{2}+\left\|\nabla u_{0}\right\|_{L^{2}\left(\Omega_{1}\right)}^{2}-\mathrm{i} \mu\left\|\nabla v_{0}\right\|_{L^{2}\left(\Omega_{2}\right)}^{2}+\mathrm{i} \mu \mu^{3}\left\|v_{0}\right\|_{L^{2}\left(\Omega_{2}\right)}^{2}+\int_{\gamma} \partial_{n^{\prime}} v_{0} \bar{g}_{0} \mathrm{~d} \sigma .
\end{aligned}
$$

Taking the real part of this expression, we get

$$
\left\|\nabla u_{0}\right\|_{L^{2}\left(\Omega_{1}\right)}^{2} \leq\left\|(\triangle-\mathrm{i} \mu) u_{0}\right\|_{L^{2}\left(\Omega_{1}\right)}\left\|u_{0}\right\|_{L^{2}\left(\Omega_{1}\right)}+\left\|\left(\triangle+\mu^{2}\right) v_{0}\right\|_{L^{2}\left(\Omega_{2}\right)}\left\|v_{0}\right\|_{L^{2}\left(\Omega_{2}\right)}+\left|\int_{\gamma} \partial_{n^{\prime}} v_{0} \bar{g}_{0} \mathrm{~d} \sigma\right| .
$$

Recalling that $\triangle v_{0}=g_{1}+\mathrm{i} \mu g_{0}-\mu^{2} v_{0}$ and using the trace lemma (Lem. 3.4 in [6]), we obtain

$$
\left\|\partial_{n} v_{0}\right\|_{H^{-\frac{1}{2}}(\gamma)} \leq C\left(\mu^{2}\left\|v_{0}\right\|_{H^{1}\left(\Omega_{2}\right)}+\left\|g_{1}+\mathrm{i} \mu g_{0}\right\|_{L^{2}\left(\Omega_{2}\right)}\right) .
$$

Combining with (2.7), we obtain

$$
\begin{aligned}
\left\|\nabla u_{0}\right\|_{L^{2}\left(\Omega_{1}\right)}^{2} \leq\left\|f_{0}\right\|_{L^{2}\left(\Omega_{1}\right)}\left\|u_{0}\right\|_{L^{2}\left(\Omega_{1}\right)}+\left\|g_{1}+\mathrm{i} \mu g_{0}\right\|_{L^{2}\left(\Omega_{2}\right)} & \left\|v_{0}\right\|_{L^{2}\left(\Omega_{2}\right)} \\
& +\left(\mu^{2}\left\|v_{0}\right\|_{H^{1}\left(\Omega_{2}\right)}+\left\|g_{1}+\mathrm{i} \mu g_{0}\right\|_{L^{2}\left(\Omega_{2}\right)}\right)\left\|g_{0}\right\|_{H^{\frac{1}{2}}(\gamma)} .
\end{aligned}
$$

Then

$$
\begin{aligned}
\left\|\nabla u_{0}\right\|_{L^{2}\left(\Omega_{1}\right)}^{2} \leq \frac{C}{\epsilon}\left\|f_{0}\right\|_{L^{2}\left(\Omega_{1}\right)}^{2}+\epsilon\left\|u_{0}\right\|_{L^{2}\left(\Omega_{1}\right)}^{2}+\frac{C}{\epsilon} \| & g_{1}+\mathrm{i} \mu g_{0}\left\|_{L^{2}\left(\Omega_{2}\right)}^{2}+\epsilon\right\| v_{0} \|_{L^{2}\left(\Omega_{2}\right)}^{2} \\
& +\left(\mu^{2}\left\|v_{0}\right\|_{H^{1}\left(\Omega_{2}\right)}+\left\|g_{1}+\mathrm{i} \mu g_{0}\right\|_{L^{2}\left(\Omega_{2}\right)}\right)\left\|g_{0}\right\|_{H^{\frac{1}{2}}(\gamma)} .
\end{aligned}
$$

Now we use this result proved in Appendix A. 
Lemma 2.1. Let $\mathcal{O}$ be a bounded open set of $\mathbb{R}^{n}$. Then for all $\mu_{0}>0$, there exists $C>0$ such that for $u$ and $f$ satisfying $(\triangle-\mathrm{i} \mu) u=f$ in $\mathcal{O},|\mu| \geq \mu_{0}$, we have the following estimate

$$
\|u\|_{H^{1}(\mathcal{O})} \leq C\left(\|\nabla u\|_{L^{2}(\mathcal{O})}+\|f\|_{L^{2}(\mathcal{O})}\right)
$$

Using this lemma and (2.8), we obtain, for $\epsilon$ small enough

$$
\begin{aligned}
\left\|u_{0}\right\|_{H^{1}\left(\Omega_{1}\right)}^{2} \leq C\left\|f_{0}\right\|_{L^{2}\left(\Omega_{1}\right)}^{2}+C_{\epsilon}\left\|g_{1}+\mathrm{i} \mu g_{0}\right\|_{L^{2}\left(\Omega_{2}\right)}^{2}+\epsilon\left\|v_{0}\right\|_{L^{2}\left(\Omega_{2}\right)}^{2} \\
+\left(\mu^{2}\left\|v_{0}\right\|_{H^{1}\left(\Omega_{2}\right)}+\left\|g_{1}+\mathrm{i} \mu g_{0}\right\|_{L^{2}\left(\Omega_{2}\right)}\right)\left\|g_{0}\right\|_{H^{\frac{1}{2}}(\gamma)}
\end{aligned}
$$

Then there exists $c_{3} \gg c_{1}$ such that

$$
\left\|u_{0}\right\|_{H^{1}\left(\Omega_{1}\right)}^{2} \leq C\left(\left\|f_{0}\right\|_{L^{2}\left(\Omega_{1}\right)}^{2}+\epsilon \mathrm{e}^{-c_{3}|\mu|}\left\|v_{0}\right\|_{H^{1}\left(\Omega_{2}\right)}^{2}+C_{\epsilon} \mathrm{e}^{-c_{3}|\mu|}\left\|g_{1}+\mathrm{i} \mu g_{0}\right\|_{L^{2}\left(\Omega_{2}\right)}^{2}+\mathrm{e}^{c_{3}|\mu|}\left\|g_{0}\right\|_{H^{1}\left(\Omega_{2}\right)}^{2}\right) .
$$

Plugging (2.10) in (2.4), we obtain, for $\epsilon$ small enough

$$
\left\|v_{0}\right\|_{H^{1}\left(\Omega_{2}\right)}^{2} \leq C \mathrm{e}^{c|\mu|}\left(\left\|f_{0}\right\|_{L^{2}\left(\Omega_{1}\right)}^{2}+\left\|g_{0}\right\|_{H^{1}\left(\Omega_{2}\right)}^{2}+\left\|g_{1}+\mathrm{i} \mu g_{0}\right\|_{L^{2}\left(\Omega_{2}\right)}^{2}\right)
$$

Combining (2.10) and (2.11), we obtain

$$
\left\|u_{0}\right\|_{H^{1}\left(\Omega_{1}\right)}^{2} \leq C \mathrm{e}^{c|\mu|}\left(\left\|f_{0}\right\|_{L^{2}\left(\Omega_{1}\right)}^{2}+\left\|g_{0}\right\|_{H^{1}\left(\Omega_{2}\right)}^{2}+\left\|g_{1}+\mathrm{i} \mu g_{0}\right\|_{L^{2}\left(\Omega_{2}\right)}^{2}\right)
$$

Recalling that $v_{1}=g_{0}+\mathrm{i} \mu v_{0}$ and using (2.11), we obtain

$$
\left\|v_{1}\right\|_{H^{1}\left(\Omega_{2}\right)}^{2} \leq C \mathrm{e}^{c|\mu|}\left(\left\|f_{0}\right\|_{L^{2}\left(\Omega_{1}\right)}^{2}+\left\|g_{0}\right\|_{H^{1}\left(\Omega_{2}\right)}^{2}+\left\|g_{1}+\mathrm{i} \mu g_{0}\right\|_{L^{2}\left(\Omega_{2}\right)}^{2}\right) .
$$

Combining (2.11), (2.12) and (2.13), we obtain Theorem 1.1.

Proof of Theorem 2.1. Estimate (2.4) is the consequence of two important results. The first one is a known result proved by Lebeau and Robbiano in [10] and the second one is given by Theorem 2.2 and proved in Section 3.

Let $0<\epsilon_{1}<\epsilon_{2}$ and $V_{\epsilon_{j}}, j=1,2$, such that $V_{\epsilon_{j}}=\left\{x \in \Omega_{2}, d(x, \gamma)<\epsilon_{j}\right\}$.

Recalling that $\left(\triangle+\mu^{2}\right) v_{0}=g_{1}+\mathrm{i} \mu g_{0}$, then for all $D>0$, there exists $C>0$ and $\left.\nu \in\right] 0,1[$ such that we have the following estimate (see [10])

$$
\left\|v_{0}\right\|_{H^{1}\left(\Omega_{2} \backslash V_{\epsilon_{1}}\right)} \leq C \mathrm{e}^{D|\mu|}\left\|v_{0}\right\|_{H^{1}\left(\Omega_{2}\right)}^{1-\nu}\left(\left\|g_{1}+\mathrm{i} \mu g_{0}\right\|_{L^{2}\left(\Omega_{2}\right)}+\left\|v_{0}\right\|_{H^{1}\left(V_{\epsilon_{2}}\right)}\right)^{\nu} .
$$

Moreover we have the following result proved in Section 3.

Theorem 2.2. There exist $C>0, \epsilon_{2}>0$ and $\mu_{0}>0$ such that for any $|\mu| \geq \mu_{0}$, for all $k_{2}>0$, there exists $k_{1}>0$ such that we have the following estimate

$$
\begin{aligned}
\left\|v_{0}\right\|_{H^{1}\left(V_{\epsilon_{2}}\right)}^{2} \leq & C \mathrm{e}^{k_{1}|\mu|}\left[\left\|f_{0}\right\|_{L^{2}\left(\Omega_{1}\right)}^{2}+\left\|g_{1}+\mathrm{i} \mu g_{0}\right\|_{L^{2}\left(\Omega_{2}\right)}^{2}+\left\|g_{0}\right\|_{H^{1}\left(\Omega_{2}\right)}^{2}+\left\|u_{0}\right\|_{H^{1}\left(\Omega_{1}\right)}^{2}\right] \\
& +C \mathrm{e}^{-k_{2}|\mu|}\left\|v_{0}\right\|_{H^{1}\left(\Omega_{2}\right)}^{2} .
\end{aligned}
$$


Combining (2.14) and (2.15), we obtain

$$
\begin{aligned}
\left\|v_{0}\right\|_{H^{1}\left(\Omega_{2} \backslash V_{\epsilon_{2}}\right) \leq}^{2} \leq & C \epsilon\left\|v_{0}\right\|_{H^{1}\left(\Omega_{2}\right)}^{2}+\frac{C}{\epsilon^{\frac{1-\nu}{\nu}}} \mathrm{e}^{2 \frac{D}{\nu}|\mu|}\left\|g_{1}+\mathrm{i} \mu g_{0}\right\|_{L^{2}\left(\Omega_{2}\right)}^{2}+\frac{C}{\epsilon^{\frac{1-\nu}{\nu}}} \mathrm{e}^{\left(2 \frac{D}{\nu}-k_{2}\right)|\mu|}\left\|v_{0}\right\|_{H^{1}\left(\Omega_{2}\right)}^{2} \\
& +\frac{C}{\epsilon^{\frac{1-\nu}{\nu}}} \mathrm{e}^{\left(2 \frac{D}{\nu}+k_{1}\right)|\mu|}\left[\left\|f_{0}\right\|_{L^{2}\left(\Omega_{1}\right)}^{2}+\left\|g_{1}+\mathrm{i} \mu g_{0}\right\|_{L^{2}\left(\Omega_{2}\right)}^{2}+\left\|g_{0}\right\|_{H^{1}\left(\Omega_{2}\right)}^{2}+\left\|u_{0}\right\|_{H^{1}\left(\Omega_{1}\right)}^{2}\right] .
\end{aligned}
$$

Adding (2.15) and (2.16), we obtain

$$
\begin{aligned}
\left\|v_{0}\right\|_{H^{1}\left(\Omega_{2}\right)}^{2} \leq C \epsilon\left\|v_{0}\right\|_{H^{1}\left(\Omega_{2}\right)}^{2}+C_{\epsilon} \mathrm{e}^{2 \frac{D}{\nu}}\left\|g_{1}+\mathrm{i} \mu g_{0}\right\|_{L^{2}\left(\Omega_{2}\right)}^{2}+C_{\epsilon} \mathrm{e}^{\left(2 \frac{D}{\nu}-k_{2}\right)|\mu|}\left\|v_{0}\right\|_{H^{1}\left(\Omega_{2}\right)}^{2} \\
+C_{\epsilon} \mathrm{e}^{\left(2 \frac{D}{\nu}+k_{1}\right)|\mu|}\left[\left\|f_{0}\right\|_{L^{2}\left(\Omega_{1}\right)}^{2}+\left\|g_{1}+\mathrm{i} \mu g_{0}\right\|_{L^{2}\left(\Omega_{2}\right)}^{2}+\left\|g_{0}\right\|_{H^{1}\left(\Omega_{2}\right)}^{2}+\left\|u_{0}\right\|_{H^{1}\left(\Omega_{1}\right)}^{2}\right] .
\end{aligned}
$$

We fix $\epsilon$ small enough and $k_{2}$ such that $2 \frac{D}{\nu} \ll k_{2}$, then there exists $\mu_{0}>0$ such that for any $|\mu| \geq \mu_{0}$, we obtain (2.4).

\section{The Carleman estimate and its consequence}

In this part, we prove the new Carleman estimate and Theorem 2.2 which is a consequence of this estimate.

\subsection{Statement of the Carleman estimate}

In this subsection we state the Carleman estimate which is the starting point of the proof of the main result. We begin by giving some notations and definitions used in the sequel.

Let $\tau$ be a positive real number such that $\tau \geq C_{0}|\mu|, C_{0}>0$. We define the Sobolev spaces with a parameter $\tau$, $H_{\tau}^{s}$ by

$$
u(x, \tau) \in H_{\tau}^{s} \quad \Longleftrightarrow \quad\langle\xi, \tau\rangle^{s} \widehat{u}(\xi, \tau) \in L^{2}, \quad\langle\xi, \tau\rangle^{2}=|\xi|^{2}+\tau^{2},
$$

where $\widehat{u}$ denoted the partial Fourier transform with respect to $x$.

For a differential operator

$$
P(x, D, \tau, \mu)=\sum_{|\alpha|+k+j \leq m} a_{\alpha, k}(x) \mu^{k} \tau^{j} D^{\alpha},
$$

we denote the associated symbol by

$$
p(x, \xi, \tau, \mu)=\sum_{|\alpha|+k+j \leq m} a_{\alpha, k}(x) \mu^{k} \tau^{j} \xi^{\alpha} .
$$

The class of symbols of order $m$ is defined by

$$
S_{\tau}^{m}=\left\{p(x, \xi, \tau, \mu) \in C^{\infty},\left|D_{x}^{\alpha} D_{\xi}^{\beta} p(x, \xi, \tau, \mu)\right| \leq C_{\alpha, \beta}\langle\xi, \tau\rangle^{m-|\beta|}\right\}
$$

and the class of tangential symbols of order $m$ by

$$
\mathcal{T} S_{\tau}^{m}=\left\{p\left(x, \xi^{\prime}, \tau, \mu\right) \in C^{\infty},\left|D_{x}^{\alpha} D_{\xi^{\prime}}^{\beta} p\left(x, \xi^{\prime}, \tau, \mu\right)\right| \leq C_{\alpha, \beta}\left\langle\xi^{\prime}, \tau\right\rangle^{m-|\beta|}\right\} .
$$

We denote by $\mathcal{O}^{m}\left(\right.$ resp. $\left.\mathcal{T} \mathcal{O}^{m}\right)$ the set of pseudo-differential operators $P=\operatorname{op}(p), p \in S_{\tau}^{m}$ (resp. $\left.\mathcal{T} S_{\tau}^{m}\right)$ and by $\sigma(P)$ the principal symbol of $P$.

We shall frequently use the symbol $\Lambda=\left\langle\xi^{\prime}, \tau\right\rangle=\left(\left|\xi^{\prime}\right|^{2}+\tau^{2}\right)^{\frac{1}{2}}$.

We use the following Gårding estimate: if $p \in \mathcal{T} S_{\tau}^{2}$ satisfies for $C_{0}>0, p\left(x, \xi^{\prime}, \tau\right)+\bar{p}\left(x, \xi^{\prime}, \tau\right) \geq C_{0} \Lambda^{2}$, then

$$
\exists C_{1}>0, \exists \tau_{0}>0, \forall \tau>\tau_{0}, \forall u \in C_{0}^{\infty}(K), \operatorname{Re}\left(P\left(x, D^{\prime}, \tau, \mu\right) u, u\right) \geq C_{1}\|\operatorname{op}(\Lambda) u\|_{L^{2}}^{2} .
$$


Let $u=\left(u_{0}, v_{0}\right)$ satisfy the equation

$$
\begin{cases}-(\triangle-\mathrm{i} \mu) u_{0}=f_{1} & \text { in } \Omega_{1}, \\ -\left(\triangle+\mu^{2}\right) v_{0}=f_{2} & \text { in } \Omega_{2}, \\ \text { op }\left(B_{1}\right) u=u_{0}-\mathrm{i} \mu v_{0}=e_{1} & \text { on } \gamma \\ \mathrm{op}\left(B_{2}\right) u=\partial_{n} u_{0}-\partial_{n} v_{0}=e_{2} & \text { on } \gamma\end{cases}
$$

We will proceed like Bellassoued in [3], we will reduce the problem of transmission to a diagonal system defined only on one side of the interface with boundary conditions.

Let $x=\left(x^{\prime}, x_{n}\right) \in \mathbb{R}^{n-1} \times \mathbb{R}$. In a neighborhood $W \subset \mathbb{R}^{n}$ of $(0,0)$, we use normal geodesic coordinates (we can assume $W$ symmetric with respect to $\left.x_{n} \mapsto-x_{n}\right)$. We denote

$$
\Theta_{2}=\left\{x \in \mathbb{R}^{n}, x_{n}>0\right\} \cap W, \quad \text { and } \quad \Theta_{1}=\left\{x \in \mathbb{R}^{n}, x_{n}<0\right\} \cap W .
$$

The Laplacian on $\Theta_{2}$ is written in the form

$$
\triangle=-A_{2}(x, D)=-\left(D_{x_{n}}^{2}+R\left(+x_{n}, x^{\prime}, D_{x^{\prime}}\right)\right) .
$$

The Laplacian on $\Theta_{1}$ can be identified locally to an operator in $\Theta_{2}$ given by

$$
\triangle=-A_{1}(x, D)=-\left(D_{x_{n}}^{2}+R\left(-x_{n}, x^{\prime}, D_{x^{\prime}}\right)\right) .
$$

We denote the operator, with $C^{\infty}$ coefficients defined in $\Theta_{2}$, by

$$
A(x, D)=\operatorname{diag}\left(A_{1}\left(x, D_{x}\right), A_{2}\left(x, D_{x}\right)\right)
$$

and the tangential operator by

$$
R\left(x, D_{x^{\prime}}\right)=\operatorname{diag}\left(R\left(-x_{n}, x^{\prime}, D_{x^{\prime}}\right), R\left(+x_{n}, x^{\prime}, D_{x^{\prime}}\right)\right)=\operatorname{diag}\left(R_{1}\left(x, D_{x^{\prime}}\right), R_{2}\left(x, D_{x^{\prime}}\right)\right) .
$$

The principal symbol of the differential operator $A(x, D)$ satisfies $\sigma(A)=\xi_{n}^{2}+r\left(x, \xi^{\prime}\right)$, where $r\left(x, \xi^{\prime}\right)=$ $\operatorname{diag}\left(r_{1}\left(x, \xi^{\prime}\right), r_{2}\left(x, \xi^{\prime}\right)\right)=\sigma\left(R\left(x, D_{x^{\prime}}\right)\right)$ and the quadratic form $r_{j}\left(x, \xi^{\prime}\right), j=1,2$, satisfies

$$
\exists C>0, \quad \forall\left(x, \xi^{\prime}\right), \quad r_{j}\left(x, \xi^{\prime}\right) \geq C\left|\xi^{\prime}\right|^{2}, \quad j=1,2 .
$$

We denote $P(x, D)$ the matrix operator with $C^{\infty}$ coefficients defined in $\Theta_{2}$, by

$$
P(x, D)=\operatorname{diag}\left(P_{1}(x, D), P_{2}(x, D)\right)=\left(\begin{array}{cc}
A_{1}(x, D)+\mathrm{i} \mu & 0 \\
0 & A_{2}(x, D)-\mu^{2}
\end{array}\right) .
$$

Let $\varphi(x)=\operatorname{diag}\left(\varphi_{1}(x), \varphi_{2}(x)\right)$, with $\varphi_{j}, j=1,2$, are $C^{\infty}$ functions in $\Theta_{2}$. For $\tau$ large enough, we define the operator

$$
A(x, D, \tau)=\mathrm{e}^{\tau \varphi} A(x, D) \mathrm{e}^{-\tau \varphi}
$$

where the principal symbol of $A(x, D, \tau)$ is given by

$$
\sigma(A)=\left(\xi_{n}+\mathrm{i} \tau \frac{\partial \varphi}{\partial x_{n}}\right)^{2}+r\left(x, \xi^{\prime}+\mathrm{i} \frac{\partial \varphi}{\partial x^{\prime}}\right) \in S_{\tau}^{2}
$$

Let

$$
\tilde{Q}_{2, j}=\frac{1}{2}\left(A_{j}+A_{j}^{*}\right), \quad \tilde{Q}_{1, j}=\frac{1}{2 \mathrm{i}}\left(A_{j}-A_{j}^{*}\right), \quad j=1,2
$$


its real and imaginary part. Then we have

$$
\left\{\begin{array}{l}
A_{j}=\tilde{Q}_{2, j}+i \tilde{Q}_{1, j}, \\
\sigma\left(\tilde{Q}_{2, j}\right)=\xi_{n}^{2}+q_{2, j}\left(x, \xi^{\prime}, \tau\right), \quad \sigma\left(\tilde{Q}_{1, j}\right)=2 \tau \frac{\partial \varphi_{j}}{\partial x_{n}} \xi_{n}+2 \tau q_{1, j}\left(x, \xi^{\prime}, \tau\right), \quad j=1,2,
\end{array}\right.
$$

where $q_{1, j} \in \mathcal{T} S_{\tau}^{1}$ and $q_{2, j} \in \mathcal{T} S_{\tau}^{2}$ are two tangential symbols given by

$$
\left\{\begin{array}{l}
q_{2, j}\left(x, \xi^{\prime}, \tau\right)=r_{j}\left(x, \xi^{\prime}\right)-\left(\tau \frac{\partial \varphi_{j}}{\partial x_{n}}\right)^{2}-\tau^{2} r_{j}\left(x, \frac{\partial \varphi_{j}}{\partial x^{\prime}}\right) \\
q_{1, j}\left(x, \xi^{\prime}, \tau\right)=\tilde{r}_{j}\left(x, \xi^{\prime}, \frac{\partial \varphi_{j}}{\partial x^{\prime}}\right), \quad j=1,2
\end{array}\right.
$$

where $\tilde{r}\left(x, \xi^{\prime}, \eta^{\prime}\right)$ is the bilinear form associated to the quadratic form $r\left(x, \xi^{\prime}\right)$.

In the sequel, $P(x, D, \tau, \mu)$ is the matrix operator with $C^{\infty}$ coefficients defined in $\Theta_{2}$ by

$$
P(x, D, \tau, \mu)=\operatorname{diag}\left(P_{1}(x, D, \tau, \mu), P_{2}(x, D, \tau, \mu)\right)=\left(\begin{array}{cc}
A_{1}(x, D, \tau)+\mathrm{i} \mu & 0 \\
0 & A_{2}(x, D, \tau)-\mu^{2}
\end{array}\right)
$$

and $u=\left(u_{0}, v_{0}\right)$ satisfies the equation

$$
\left\{\begin{array}{lr}
P u=f & \text { in }\left\{x_{n}>0\right\} \cap W, \\
\mathrm{op}\left(b_{1}\right) u=\left.u_{0}\right|_{x_{n}=0}-\left.\mathrm{i} \mu v_{0}\right|_{x_{n}=0}=e_{1} & \text { on }\left\{x_{n}=0\right\} \cap W, \\
\mathrm{op}\left(b_{2}\right) u=\left.\left(D_{x_{n}}+\mathrm{i} \tau \frac{\partial \varphi_{1}}{\partial x_{n}}\right) u_{0}\right|_{x_{n}=0}+\left.\left(D_{x_{n}}+\mathrm{i} \tau \frac{\partial \varphi_{2}}{\partial x_{n}}\right) v_{0}\right|_{x_{n}=0}=e_{2} & \text { on }\left\{x_{n}=0\right\} \cap W,
\end{array}\right.
$$

where $f=\left(f_{1}, f_{2}\right), e=\left(e_{1}, e_{2}\right)$ and $B=\left(\operatorname{op}\left(b_{1}\right), \mathrm{op}\left(b_{2}\right)\right)$. We note $p_{j}(x, \xi, \tau, \mu), j=1,2$, the principal symbol associated to $P_{j}(x, D, \tau, \mu)$. We have

$$
\left\{\begin{array}{l}
p_{1}(x, \xi, \tau, \mu)=\xi_{n}^{2}+q_{2,1}\left(x, \xi^{\prime}, \tau\right)+\mathrm{i}\left(2 \tau \frac{\partial \varphi_{1}}{\partial x_{n}} \xi_{n}+2 \tau q_{1,1}\left(x, \xi^{\prime}, \tau\right)\right) \\
p_{2}(x, \xi, \tau, \mu)=\xi_{n}^{2}+q_{2,2}\left(x, \xi^{\prime}, \tau\right)-\mu^{2}+\mathrm{i}\left(2 \tau \frac{\partial \varphi_{2}}{\partial x_{n}} \xi_{n}+2 \tau q_{1,2}\left(x, \xi^{\prime}, \tau\right)\right) .
\end{array}\right.
$$

We assume that $\varphi$ satisfies

$$
\begin{cases}\varphi_{1}(x)=\varphi_{2}(x) & \text { on }\left\{x_{n}=0\right\} \cap W \\ \frac{\partial \varphi_{1}}{\partial x_{n}}>0 & \text { on }\left\{x_{n}=0\right\} \cap W \\ \left(\frac{\partial \varphi_{1}}{\partial x_{n}}\right)^{2}-\left(\frac{\partial \varphi_{2}}{\partial x_{n}}\right)^{2}>1 & \text { on }\left\{x_{n}=0\right\} \cap W\end{cases}
$$

and the following hypoellipticity condition of Hörmander: $\exists C>0, \forall x \in K, \forall \xi \in \mathbb{R}^{n} \backslash\{0\}$,

$$
\left(\operatorname{Re} p_{j}=0 \quad \text { et } \frac{1}{2 \tau} \operatorname{Im} p_{j}=0\right) \Rightarrow\left\{\operatorname{Re} p_{j}, \frac{1}{2 \tau} \operatorname{Im} p_{j}\right\} \geq C\langle\xi, \tau\rangle^{2},
$$

where $\{f, g\}(x, \xi)=\sum\left(\frac{\partial f}{\partial \xi_{j}} \frac{\partial g}{\partial x_{j}}-\frac{\partial f}{\partial x_{j}} \frac{\partial g}{\partial \xi_{j}}\right)$ is the Poisson bracket of two functions $f(x, \xi)$ and $g(x, \xi)$ and $K$ is a compact in $\left\{x_{n} \geq 0\right\} \cap W$. 
We denote by

$$
\begin{gathered}
\|u\|_{L^{2}\left(\Theta_{2}\right)}=\|u\|, \quad\|u\|_{k, \tau}^{2}=\sum_{j=0}^{k} \tau^{2(k-j)}\|u\|_{H^{j}\left(\Theta_{2}\right)}^{2}, \quad\|u\|_{k}^{2}=\left\|\operatorname{op}\left(\Lambda^{k}\right) u\right\|^{2}, \\
|u|_{k, \tau}^{2}=\left\|\left.u\right|_{x_{n}=0}\right\|_{k, \tau}^{2}, \quad|u|_{k}^{2}=\left\|\left.u\right|_{x_{n}=0}\right\|_{k}^{2}, k \in \mathbb{R} \quad \text { and } \quad|u|_{1,0, \tau}^{2}=|u|_{1}^{2}+\left|D_{x_{n}} u\right|^{2} .
\end{gathered}
$$

We are now ready to state our result.

Theorem 3.1. Let $\varphi$ satisfies (3.8) and (3.9). Then there exist constants $C>0, \tau_{0}>0$ and $\mu_{0}>0$ such that for any $\tau \geq \tau_{0},|\mu| \geq \mu_{0}$ such that $\tau \geq C_{0}|\mu|, C_{0}>0$, we have the following estimate

$$
\tau\|w\|_{1, \tau}^{2}+\tau^{2}|w|_{\frac{1}{2}}^{2}+\tau^{2}\left|D_{x_{n}} w\right|_{-\frac{1}{2}}^{2} \leq C\left(\|P(x, D, \tau) w\|^{2}+\frac{\tau^{2}}{\mu^{2}}\left|\operatorname{op}\left(b_{1}\right) w\right|_{\frac{1}{2}}^{2}+\tau\left|\operatorname{op}\left(b_{2}\right) w\right|^{2}\right)
$$

for any $w \in C_{0}^{\infty}\left(\left\{x_{n} \geq 0\right\} \cap W\right)$.

Corollary 3.1. Let $\varphi$ satisfies (3.8) and (3.9). Then there exist constants $C>0, \tau_{0}>0$ and $\mu_{0}>0$ such that for any $\tau \geq \tau_{0},|\mu| \geq \mu_{0}$ such that $\tau \geq C_{0}|\mu|, C_{0}>0$, we have the following estimate

$$
\mu\left\|\mathrm{e}^{\mu \varphi} h\right\|_{H^{1}\left(\Theta_{2}\right)}^{2} \leq C\left(\left\|\mathrm{e}^{\mu \varphi} P(x, D) h\right\|_{L^{2}\left(\Theta_{2}\right)}^{2}+\frac{\tau^{2}}{\mu^{2}}\left\|\mathrm{e}^{\mu \varphi} \mathrm{op}\left(B_{1}\right) h\right\|_{H^{\frac{1}{2}\left(x_{n}=0\right)}}^{2}+\mu\left\|\mathrm{e}^{\mu \varphi} \mathrm{op}\left(B_{2}\right) h\right\|_{L^{2}\left(x_{n}=0\right)}^{2}\right)
$$

for any $h \in C_{0}^{\infty}\left(\left\{x_{n} \geq 0\right\} \cap W\right)$.

Proof. Let $w=\mathrm{e}^{\tau \varphi} h$. Recalling that $P(x, D, \tau, \mu) w=\mathrm{e}^{\tau \varphi} P(x, D) \mathrm{e}^{-\tau \varphi} w$ and using (3.10), we obtain (3.11).

\subsection{Proof of Theorem $\mathbf{2 . 2}$}

To apply Corollary 3.1, we have to choose $\varphi_{1}$ and $\varphi_{2}$ satisfying (3.8) and (3.9).

We denote $x=\left(x^{\prime}, x_{n}\right)$ a point in $\Omega$. Let $x_{0}=(0,-\delta), \delta>0$. We set

$$
\begin{gathered}
\psi(x)=\left|x-x_{0}\right|^{2}-\delta^{2} \quad \text { and } \\
\varphi_{1}(x)=\mathrm{e}^{-\beta \psi\left(x^{\prime},-x_{n}\right)}, \quad \varphi_{2}(x)=\mathrm{e}^{-\beta\left(\psi(x)-\alpha x_{n}\right)}, \quad \beta>0, \quad \text { and } \quad \frac{\delta}{2}<\alpha<2 \delta .
\end{gathered}
$$

The weight function $\varphi=\operatorname{diag}\left(\varphi_{1}, \varphi_{2}\right)$ has to satisfy (3.8) and (3.9). With these choices, we have $\left.\varphi_{1}\right|_{x_{n}=0}=$ $\left.\varphi_{2}\right|_{x_{n}=0}$ and $\left.\frac{\partial \varphi_{1}}{\partial x_{n}}\right|_{x_{n}=0}>0$. It remains to verify

$$
\left(\frac{\partial \varphi_{1}}{\partial x_{n}}\right)^{2}-\left(\frac{\partial \varphi_{2}}{\partial x_{n}}\right)^{2}>1 \text { on }\left\{x_{n}=0\right\}
$$

and the condition (3.9). We begin by condition (3.9) and we compute for $\varphi_{1}$ and $p_{1}$ (the computation for $\varphi_{2}$ and $p_{2}$ is made in the same way). Recalling that

$$
\begin{aligned}
\left\{\operatorname{Re} p_{1}, \frac{1}{2 \tau} \operatorname{Im} p_{1}\right\}(x, \xi)= & \frac{\operatorname{Im}}{2 \tau}\left[\partial_{\xi} p_{1}\left(x, \xi-\mathrm{i} \tau \varphi_{1}^{\prime}(x)\right) \partial_{x} p_{1}\left(x, \xi+\mathrm{i} \tau \varphi_{1}^{\prime}(x)\right)\right] \\
& +{ }^{t}\left[\partial_{\xi} p_{1}\left(x, \xi-\mathrm{i} \tau \varphi_{1}^{\prime}(x)\right)\right] \varphi_{1}^{\prime \prime}(x)\left[\partial_{\xi} p_{1}\left(x, \xi-\mathrm{i} \tau \varphi_{1}^{\prime}(x)\right)\right] .
\end{aligned}
$$


We replace $\varphi_{1}(x)$ by $\varphi_{1}(x)=\mathrm{e}^{-\beta \psi\left(x^{\prime},-x_{n}\right)}, \beta>0$, we obtain, by noting $\xi=-\beta \varphi_{1}(x) \eta$

$$
\begin{gathered}
\left\{\operatorname{Re} p_{1}, \frac{1}{2 \tau} \operatorname{Im} p_{1}\right\}(x, \xi)= \\
\left(-\beta \varphi_{1}\right)^{3}\left[\left\{\operatorname{Re} p_{1}\left(x, \eta-\mathrm{i} \tau \psi^{\prime}\right), \frac{1}{2 \tau} \operatorname{Im} p_{1}\left(x, \eta+\mathrm{i} \tau \psi^{\prime}\right)\right\}(x, \eta)\right. \\
\left.-\beta\left|\psi^{\prime}(x) \partial_{\eta} p_{1}\left(x, \eta+\mathrm{i} \tau \psi^{\prime}\right)\right|^{2}\right]
\end{gathered}
$$

and

$$
\left|\psi^{\prime}(x) \partial_{\eta} p_{1}\left(x, \eta+\mathrm{i} \tau \psi^{\prime}\right)\right|^{2}=4\left[\tau^{2}\left|p_{1}\left(x, \psi^{\prime}\right)\right|^{2}+\left|\tilde{p}_{1}\left(x, \eta, \psi^{\prime}\right)\right|^{2}\right]
$$

where $\tilde{p}_{1}\left(x, \eta, \psi^{\prime}\right)$ is the bilinear form associated to the quadratic form $p_{1}(x, \eta)$. We have

$$
\left(\operatorname{Re} p_{1}=0 \quad \text { et } \quad \frac{1}{2 \tau} \operatorname{Im} p_{1}=0\right) \Longleftrightarrow p_{1}\left(x, \eta+\mathrm{i} \tau \psi^{\prime}\right)=0
$$

- If $\tau=0$, we have $p_{1}(x, \xi)=0$ which is impossible. Indeed, we have $p_{1}(x, \xi) \geq C|\xi|^{2}, \forall(x, \xi) \in K \times \mathbb{R}^{n}, K$ compact in $\left\{x_{n} \geq 0\right\} \cap W$.

- If $\tau \neq 0$, we have $\tilde{p}_{1}\left(x, \eta, \psi^{\prime}\right)=0$.

Then $\left|\psi^{\prime}(x) \partial_{\eta} p_{1}\left(x, \eta+\mathrm{i} \tau \psi^{\prime}\right)\right|^{2}=4 \tau^{2}\left|p_{1}\left(x, \psi^{\prime}\right)\right|^{2}>0$. On the other hand, we have

$$
\left\{\operatorname{Re} p_{1}\left(x, \eta-\mathrm{i} \tau \psi^{\prime}\right), \frac{1}{2 \tau} \operatorname{Im} p_{1}\left(x, \eta+\mathrm{i} \tau \psi^{\prime}\right)\right\}(x, \eta) \leq C_{1}\left(|\eta|^{2}+\tau^{2}\left|\psi^{\prime}\right|^{2}\right)
$$

where $C_{1}$ is a positive constant independent of $\psi^{\prime}$. Then for $\beta \geq C_{1}$, the condition (3.9) is satisfied.

Now let us verify (3.12). We have, on $\left\{x_{n}=0\right\}$,

$$
\left(\frac{\partial \varphi_{1}}{\partial x_{n}}\right)^{2}-\left(\frac{\partial \varphi_{2}}{\partial x_{n}}\right)^{2}=\beta^{2} \alpha(4 \delta-\alpha) \mathrm{e}^{-2 \beta \psi} .
$$

Then to satisfy (3.12), it suffices to choose $\beta=\frac{M}{\delta}$ where $M>0$ such that $\frac{M}{\delta} \geq C_{1}$.

In the sequel, to respect the geometry we return in $\Theta_{1}$ for the heat component $u_{0}$ (in this case $\varphi_{1}$ defined above becomes $\left.\varphi_{1}(x)=\mathrm{e}^{-\beta \psi(x)}\right)$.

Let us choose $r_{1}<r_{1}^{\prime}<r_{2}<0=\psi(0)<r_{2}^{\prime}<r_{3}<r_{3}^{\prime}$. We denote

$$
w_{j}=\left\{x \in \Omega, r_{j}<\psi(x)<r_{j}^{\prime}\right\} \quad \text { and } \quad T_{x_{0}}=w_{2} \cap \Theta_{2} .
$$

We set $R_{j}=\mathrm{e}^{-\beta r_{j}}, R_{j}^{\prime}=\mathrm{e}^{-\beta r_{j}^{\prime}}, j=1,2,3$.

Then $R_{3}^{\prime}<R_{3}<R_{2}^{\prime}<R_{2}<R_{1}^{\prime}<R_{1}$. We introduce a cut-off function $\tilde{\chi} \in C_{0}^{\infty}\left(\mathbb{R}^{n+1}\right)$ such that

$$
\tilde{\chi}(\rho)=\left\{\begin{array}{ccc}
0 & \text { if } & \rho \leq r_{1}, \quad \rho \geq r_{3}^{\prime} \\
1 & \text { if } & \rho \in\left[r_{1}^{\prime}, r_{3}\right] .
\end{array}\right.
$$

Let $\tilde{u}=\left(\tilde{u}_{0}, \tilde{v}_{0}\right)=\tilde{\chi} u=\left(\tilde{\chi} u_{0}, \tilde{\chi} v_{0}\right)$, we get the following system

$$
\left\{\begin{aligned}
(\triangle-\mathrm{i} \mu) \tilde{u}_{0} & =\tilde{\chi} f_{0}+[\triangle-\mathrm{i} \mu, \tilde{\chi}] u_{0} \\
\left(\triangle+\mu^{2}\right) \tilde{v}_{0} & =\tilde{\chi}\left(g_{1}+\mathrm{i} \mu g_{0}\right)+\left[\triangle+\mu^{2}, \tilde{\chi}\right] v_{0}, \\
\tilde{v}_{1} & =g_{0}+\mathrm{i} \mu \tilde{v}_{0},
\end{aligned}\right.
$$


with the boundary conditions

$$
\begin{cases}\left.\tilde{u}_{0}\right|_{\Gamma_{1}}=\left.\tilde{v}_{0}\right|_{\Gamma_{2}} & =0 \\ \operatorname{op}\left(B_{1}\right) \tilde{u}=\tilde{u}_{0}-\mathrm{i} \mu \tilde{v}_{0} & =\left.\left(\tilde{\chi} g_{0}\right)\right|_{\gamma} \\ \operatorname{op}\left(B_{2}\right) \tilde{u} & =\left.\left(\left[\partial_{n}, \tilde{\chi}\right] u_{0}-\left[\partial_{n}, \tilde{\chi}\right] v_{0}\right)\right|_{\gamma}\end{cases}
$$

From the Carleman estimate of Corollary 3.1, we have

$$
\begin{aligned}
\tau\left\|\mathrm{e}^{\tau \varphi} \tilde{u}\right\|_{H^{1}}^{2} \leq & C\left(\left\|\mathrm{e}^{\tau \varphi_{1}}(\triangle-\mathrm{i} \mu) \tilde{u}_{0}\right\|_{L^{2}\left(\Theta_{1}\right)}^{2}+\left\|\mathrm{e}^{\tau \varphi_{2}}\left(\triangle+\mu^{2}\right) \tilde{v}_{0}\right\|_{L^{2}\left(\Theta_{2}\right)}^{2}\right. \\
& \left.+\frac{\tau^{2}}{\mu^{2}}\left\|\mathrm{e}^{\tau \varphi} \mathrm{op}\left(b_{1}\right) \tilde{u}\right\|_{H^{\frac{1}{2}}\left(x_{n}=0\right)}^{2}+\tau\left\|\mathrm{e}^{\tau \varphi} \mathrm{op}\left(b_{2}\right) \tilde{u}\right\|_{L^{2}\left(x_{n}=0\right)}^{2}\right) .
\end{aligned}
$$

Using the fact that $[\triangle-\mathrm{i} \mu, \tilde{\chi}]$ is the first order operator supported in $\left(w_{1} \cup w_{3}\right) \cap \Theta_{1}$, we have

$$
\left\|\mathrm{e}^{\tau \varphi_{1}}(\triangle-\mathrm{i} \mu) \tilde{u}_{0}\right\|_{L^{2}\left(\Theta_{1}\right)}^{2} \leq C\left(\mathrm{e}^{2 \tau R_{1}}\left\|f_{0}\right\|_{L^{2}\left(\Omega_{1}\right)}^{2}+\mathrm{e}^{2 \tau R_{1}}\left\|u_{0}\right\|_{H^{1}\left(\Omega_{1}\right)}^{2}\right)
$$

Recalling that $\left[\triangle+\mu^{2}, \tilde{\chi}\right]$ is the first order operator supported in $\left(w_{1} \cup w_{3}\right) \cap \Theta_{2}$, we show

$$
\left\|\mathrm{e}^{\tau \varphi_{2}}\left(\triangle+\mu^{2}\right) \tilde{v}_{0}\right\|_{L^{2}\left(\Theta_{2}\right)}^{2} \leq C\left(\mathrm{e}^{2 \tau}\left\|g_{1}+\mathrm{i} \mu g_{0}\right\|_{L^{2}\left(\Omega_{2}\right)}^{2}+\mathrm{e}^{2 \tau R_{3}}\left\|v_{0}\right\|_{H^{1}\left(\Omega_{2}\right)}^{2}\right) .
$$

From the trace formula and recalling that $\operatorname{op}\left(b_{2}\right) \tilde{u}$ is an operator of order zero and supported in $\left\{x_{n}=0\right\} \cap w_{3}$, we show

$$
\tau\left\|\mathrm{e}^{\tau \varphi} \mathrm{op}\left(b_{2}\right) \tilde{u}\right\|_{L^{2}\left(x_{n}=0\right)}^{2} \leq C \mathrm{e}^{2 \tau R_{3}}\|u\|_{H^{1}(\Omega)}^{2} \leq C\left(\mathrm{e}^{2 \tau R_{3}}\left\|u_{0}\right\|_{H^{1}\left(\Omega_{1}\right)}^{2}+\mathrm{e}^{2 \tau R_{3}}\left\|v_{0}\right\|_{H^{1}\left(\Omega_{2}\right)}^{2}\right) .
$$

Now we need to use this result shown in Appendix B.

Lemma 3.1. There exists $C>0$ such that for all $s \in \mathbb{R}$ and $u \in C_{0}^{\infty}(\Omega)$, we have

$$
\left\|\operatorname{op}\left(\Lambda^{s}\right) \mathrm{e}^{\tau \varphi} u\right\| \leq C \mathrm{e}^{\tau C}\left\|\operatorname{op}\left(\Lambda^{s}\right) u\right\|
$$

Following this lemma and recalling that $\tau \geq C_{0}|\mu|, C_{0}>0$, and $|\mu| \geq \mu_{0}$, we obtain

$$
\frac{\tau^{2}}{\mu^{2}}\left\|\mathrm{e}^{\tau \varphi} \mathrm{op}\left(b_{1}\right) \tilde{u}\right\|_{H^{\frac{1}{2}}\left(x_{n}=0\right)}^{2} \leq C \tau^{2} \mathrm{e}^{2 \tau c}\left|g_{0}\right|_{H^{\frac{1}{2}}}^{2} \leq C \tau^{2} \mathrm{e}^{2 \tau c}\left\|g_{0}\right\|_{H^{1}\left(\Omega_{2}\right)}^{2} .
$$

Combining (3.13)-(3.16) and (3.18), we obtain

$$
\begin{gathered}
C \tau \mathrm{e}^{2 \tau R_{2}^{\prime}}\left\|u_{0}\right\|_{H^{1}\left(w_{2} \cap \Theta_{1}\right)}^{2}+C \tau \mathrm{e}^{2 \tau R_{2}^{\prime}}\left\|v_{0}\right\|_{H^{1}\left(T_{x_{0}}\right)}^{2} \leq C\left(\mathrm{e}^{2 \tau R_{1}}\left\|f_{0}\right\|_{L^{2}\left(\Omega_{1}\right)}^{2}+\mathrm{e}^{2 \tau R_{1}}\left\|u_{0}\right\|_{H^{1}\left(\Omega_{1}\right)}^{2}\right) \\
\left.+\mathrm{e}^{2 \tau}\left\|g_{1}+\mathrm{i} \mu g_{0}\right\|_{L^{2}\left(\Omega_{2}\right)}^{2}+\mathrm{e}^{2 \tau R_{3}}\left\|v_{0}\right\|_{H^{1}\left(\Omega_{2}\right)}^{2}+\mathrm{e}^{2 \tau R_{3}}\left\|u_{0}\right\|_{H^{1}\left(\Omega_{1}\right)}^{2}+\mathrm{e}^{2 \tau c}\left\|g_{0}\right\|_{H^{1}\left(\Omega_{2}\right)}^{2}\right) .
\end{gathered}
$$

Since $R_{3}<R_{2}^{\prime}<R_{1}$. Then there exist $c_{1}>0$ and $c_{2}>0$ such that

$$
\begin{aligned}
\left\|v_{0}\right\|_{H^{1}\left(T_{x_{0}}\right)}^{2} \leq & C \mathrm{e}^{c_{1} \tau}\left[\left\|f_{0}\right\|_{L^{2}\left(\Omega_{1}\right)}^{2}+\left\|g_{1}+\mathrm{i} \mu g_{0}\right\|_{L^{2}\left(\Omega_{2}\right)}^{2}+\left\|g_{0}\right\|_{H^{1}\left(\Omega_{2}\right)}^{2}+\left\|u_{0}\right\|_{H^{1}\left(\Omega_{1}\right)}^{2}\right] \\
& +C \mathrm{e}^{-c_{2} \tau}\left\|v_{0}\right\|_{H^{1}\left(\Omega_{2}\right)}^{2} .
\end{aligned}
$$

Now we must distinguish two cases: 
- Case 1: $\left\|v_{0}\right\|_{H^{1}\left(\Omega_{2}\right)}^{2} \geq\left(\left\|f_{0}\right\|_{L^{2}\left(\Omega_{1}\right)}^{2}+\left\|g_{1}+\mathrm{i} \mu g_{0}\right\|_{L^{2}\left(\Omega_{2}\right)}^{2}+\left\|g_{0}\right\|_{H^{1}\left(\Omega_{2}\right)}^{2}+\left\|u_{0}\right\|_{H^{1}\left(\Omega_{1}\right)}^{2}\right)$

Minimizing the right-hand side of (3.19) with respect to $\tau$, we get with $\delta_{0}=c_{2} /\left(c_{1}+c_{2}\right)$, the following estimate

$$
\begin{aligned}
\left\|v_{0}\right\|_{H^{1}\left(T_{x_{0}}\right)}^{2} \leq & C\left(\left\|f_{0}\right\|_{L^{2}\left(\Omega_{1}\right)}^{2}+\left\|g_{1}+\mathrm{i} \mu g_{0}\right\|_{L^{2}\left(\Omega_{2}\right)}^{2}+\left\|g_{0}\right\|_{H^{1}\left(\Omega_{2}\right)}^{2}+\left\|u_{0}\right\|_{H^{1}\left(\Omega_{1}\right)}^{2}\right)^{\delta_{0}} \\
& \times\left(\left\|v_{0}\right\|_{H^{1}\left(\Omega_{2}\right)}^{2}\right)^{1-\delta_{0}} .
\end{aligned}
$$

- Case 2: $\left\|v_{0}\right\|_{H^{1}\left(\Omega_{2}\right)}^{2} \leq\left(\left\|f_{0}\right\|_{L^{2}\left(\Omega_{1}\right)}^{2}+\left\|g_{1}+\mathrm{i} \mu g_{0}\right\|_{L^{2}\left(\Omega_{2}\right)}^{2}+\left\|g_{0}\right\|_{H^{1}\left(\Omega_{2}\right)}^{2}+\left\|u_{0}\right\|_{H^{1}\left(\Omega_{1}\right)}^{2}\right)$

In this case (3.20) is trivial.

Then for all $k_{2}>0$, there exists $k_{1}>0$ such that we have

$$
\begin{aligned}
\left\|v_{0}\right\|_{H^{1}\left(T_{x_{0}}\right)}^{2} \leq & C \mathrm{e}^{k_{1}|\mu|}\left[\left\|f_{0}\right\|_{L^{2}\left(\Omega_{1}\right)}^{2}+\left\|g_{1}+\mathrm{i} \mu g_{0}\right\|_{L^{2}\left(\Omega_{2}\right)}^{2}+\left\|g_{0}\right\|_{H^{1}\left(\Omega_{2}\right)}^{2}+\left\|u_{0}\right\|_{H^{1}\left(\Omega_{1}\right)}^{2}\right] \\
& +C \mathrm{e}^{-k_{2}|\mu|}\left\|v_{0}\right\|_{H^{1}\left(\Omega_{2}\right)}^{2} .
\end{aligned}
$$

Since $\gamma$ is compact, then there exists a finite number of $T_{x_{0}}$ such that $\gamma \subset \cup T_{x_{0}}$ and if $\epsilon_{2}$ small enough, we have $V_{\epsilon_{2}} \subset \cup T_{x_{0}}$. Then (2.15) follows from (3.21).

\subsection{Proof of the Carleman estimate (Thm. 3.1)}

In the next section we will prove the following theorem which is analogous to Theorem 3.1 with another scale of Sobolev spaces.

Theorem 3.2. Let $\varphi$ satisfy (3.8) and (3.9). Then there exist constants $C>0, \tau_{0}>0$ and $\mu_{0}>0$ such that for any $\tau \geq \tau_{0},|\mu| \geq \mu_{0}$ such that $\tau \geq C_{0}|\mu|, C_{0}>0$, we have the following estimate

$$
\tau\|u\|_{1, \tau}^{2} \leq C\left(\|P(x, D, \tau, \mu) u\|^{2}+\tau|u|_{1,0, \tau}^{2}\right)
$$

and

$$
\tau\|u\|_{1, \tau}^{2}+\tau|u|_{1,0, \tau}^{2} \leq C\left(\|P(x, D, \tau, \mu) u\|^{2}+\frac{\tau}{\mu^{2}}\left|\operatorname{op}\left(b_{1}\right) u\right|_{1}^{2}+\tau\left|\operatorname{op}\left(b_{2}\right) u\right|^{2}\right),
$$

for any $u \in C_{0}^{\infty}\left(\left\{x_{n} \geq 0\right\} \cap W\right)$.

To prove Theorem 3.1, we need the following lemma.

Lemma 3.2. There exist constants $C>0$ and $\tau_{0}>0$ such that for any $\tau \geq \tau_{0},|\mu| \geq 1$ such that $\tau \geq C_{0}|\mu|$, $C_{0}>0$, we have the following estimate

$$
\begin{aligned}
\left\|D_{x_{n}}^{2} \mathrm{op}\left(\Lambda^{-\frac{1}{2}}\right) u\right\|^{2}+\left\|D_{x_{n}} \mathrm{op}\left(\Lambda^{\frac{1}{2}}\right) u\right\|^{2}+\left\|\operatorname{op}\left(\Lambda^{\frac{3}{2}}\right) u\right\|^{2}+\tau|u|_{1,0, \tau}^{2} \leq & C\left(\|P(x, D, \tau, \mu) u\|^{2}\right. \\
& \left.+\frac{\tau}{\mu^{2}}\left|\operatorname{op}\left(b_{1}\right) u\right|_{1}^{2}+\tau\left|\operatorname{op}\left(b_{2}\right) u\right|^{2}\right),
\end{aligned}
$$

for any $u \in C_{0}^{\infty}\left(\left\{x_{n} \geq 0\right\} \cap W\right)$.

Proof. We have

$$
P(x, D, \tau, \mu)=D_{x_{n}}^{2}+R+\tau C_{1}+\tau^{2} C_{0},
$$


where $R \in \mathcal{T} \mathcal{O}^{2}, C_{1}=c_{1}(x) D_{x_{n}}+T_{1}$, with $T_{1} \in \mathcal{T} \mathcal{O}^{1}$ and $C_{0} \in \mathcal{T} \mathcal{O}^{0}$. Then we have

$$
\left\|\left(D_{x_{n}}^{2}+R\right) \operatorname{op}\left(\Lambda^{-\frac{1}{2}}\right) u\right\|^{2} \leq C\left(\left\|\operatorname{Pop}\left(\Lambda^{-\frac{1}{2}}\right) u\right\|^{2}+\tau^{2}\left\|\mathrm{op}\left(\Lambda^{\frac{1}{2}}\right) u\right\|^{2}+\tau^{2}\left\|D_{x_{n}} \mathrm{op}\left(\Lambda^{-\frac{1}{2}}\right) u\right\|^{2}+\tau^{4}\left\|\operatorname{op}\left(\Lambda^{-\frac{1}{2}}\right) u\right\|^{2}\right)
$$

Since

$$
\begin{aligned}
& \tau^{4}\left\|\operatorname{op}\left(\Lambda^{-\frac{1}{2}}\right) u\right\|^{2} \leq C \tau^{3}\|u\|^{2}, \\
& \tau^{2}\left\|D_{x_{n}} \operatorname{op}\left(\Lambda^{-\frac{1}{2}}\right) u\right\|^{2} \leq C \tau\left\|D_{x_{n}} u\right\|^{2} \text { and } \\
& \tau^{2}\left\|\operatorname{op}\left(\Lambda^{\frac{1}{2}}\right) u\right\|^{2}=\tau^{2}\left(\frac{1}{\sqrt{\tau}} \operatorname{op}(\Lambda) u, \sqrt{\tau} u\right) \leq C\left(\tau\|\operatorname{op}(\Lambda) u\|^{2}+\tau^{3}\|u\|^{2}\right) .
\end{aligned}
$$

Using the fact that $\|u\|_{1, \tau}^{2} \simeq\|\mathrm{op}(\Lambda) u\|^{2}+\left\|D_{x_{n}} u\right\|^{2}$, we obtain

$$
\left\|\left(D_{x_{n}}^{2}+R\right) \operatorname{op}\left(\Lambda^{-\frac{1}{2}}\right) u\right\|^{2} \leq C\left(\left\|\operatorname{Pop}\left(\Lambda^{-\frac{1}{2}}\right) u\right\|^{2}+\tau\|u\|_{1, \tau}^{2}\right)
$$

Following (3.22), we have

$$
\left\|\left(D_{x_{n}}^{2}+R\right) \operatorname{op}\left(\Lambda^{-\frac{1}{2}}\right) u\right\|^{2} \leq C\left(\left\|P \operatorname{Pop}\left(\Lambda^{-\frac{1}{2}}\right) u\right\|^{2}+\|P u\|^{2}+\tau|u|_{1,0, \tau}^{2}\right) .
$$

We can write

$$
\begin{aligned}
\operatorname{Pop}\left(\Lambda^{-\frac{1}{2}}\right) u= & \operatorname{op}\left(\Lambda^{-\frac{1}{2}}\right) P u+\left[P, \operatorname{op}\left(\Lambda^{-\frac{1}{2}}\right)\right] u \\
= & \operatorname{op}\left(\Lambda^{-\frac{1}{2}}\right) P u+\left[R, \operatorname{op}\left(\Lambda^{-\frac{1}{2}}\right)\right] u \\
& +\tau\left[C_{1}, \operatorname{op}\left(\Lambda^{-\frac{1}{2}}\right)\right] u+\tau^{2}\left[C_{0}, \operatorname{op}\left(\Lambda^{-\frac{1}{2}}\right)\right] u \\
= & \operatorname{op}\left(\Lambda^{-\frac{1}{2}}\right) P u+t_{1}+t_{2}+t_{3} .
\end{aligned}
$$

Let us estimate $t_{1}, t_{2}$ and $t_{3}$. We have $\left[R, \mathrm{op}\left(\Lambda^{-\frac{1}{2}}\right)\right] \in \mathcal{T} \mathcal{O}^{\frac{1}{2}}$, then following (3.22), we have

$$
\left\|t_{1}\right\|^{2} \leq C\left\|\operatorname{op}\left(\Lambda^{\frac{1}{2}}\right) u\right\|^{2} \leq C\left(\|\operatorname{op}(\Lambda) u\|^{2}+\|u\|^{2}\right) \leq C\left(\|P u\|^{2}+\tau|u|_{1,0, \tau}^{2}\right)
$$

We have $t_{2}=\tau\left[C_{1}, \operatorname{op}\left(\Lambda^{-\frac{1}{2}}\right)\right] u=\tau\left[c_{1}(x) D_{x_{n}}, \operatorname{op}\left(\Lambda^{-\frac{1}{2}}\right)\right] u+\tau\left[T_{1}, \operatorname{op}\left(\Lambda^{-\frac{1}{2}}\right)\right] u$. Then following (3.22), we obtain

$$
\left\|t_{2}\right\|^{2} \leq C\left(\tau^{-1}\left\|D_{x_{n}} u\right\|^{2}+\tau\|u\|^{2}\right) \leq C\left(\|P u\|^{2}+\tau|u|_{1,0, \tau}^{2}\right)
$$

We have $\left[C_{0}, \operatorname{op}\left(\Lambda^{-\frac{1}{2}}\right)\right] \in \mathcal{T} \mathcal{O}^{-\frac{3}{2}}$, then following (3.22), we obtain

$$
\left\|\tau^{2}\left[C_{0}, \operatorname{op}\left(\Lambda^{-\frac{1}{2}}\right)\right] u\right\|^{2} \leq C \tau\|u\|^{2} \leq C\left(\|P u\|^{2}+\tau|u|_{1,0, \tau}^{2}\right) .
$$

From (3.26)-(3.29), we have

and from (3.25), we obtain

$$
\left\|P \operatorname{Pop}\left(\Lambda^{-\frac{1}{2}}\right) u\right\|^{2} \leq C\left(\|P u\|^{2}+\tau|u|_{1,0, \tau}^{2}\right)
$$

$$
\left\|\left(D_{x_{n}}^{2}+R\right) \operatorname{op}\left(\Lambda^{-\frac{1}{2}}\right) u\right\|^{2} \leq C\left(\|P u\|^{2}+\tau|u|_{1,0, \tau}^{2}\right) .
$$


Moreover, we have

$$
\left\|\left(D_{x_{n}}^{2}+R\right) \operatorname{op}\left(\Lambda^{-\frac{1}{2}}\right) u\right\|^{2}=\left\|D_{x_{n}}^{2} \operatorname{op}\left(\Lambda^{-\frac{1}{2}}\right) u\right\|^{2}+\left\|\operatorname{Rop}\left(\Lambda^{-\frac{1}{2}}\right) u\right\|^{2}+2 \operatorname{Re}\left(D_{x_{n}}^{2} \operatorname{op}\left(\Lambda^{-\frac{1}{2}}\right) u, \operatorname{Rop}\left(\Lambda^{-\frac{1}{2}}\right) u\right),
$$

where $(.,$.$) denote the scalar product in L^{2}$. By integration by parts, we find

$$
\begin{aligned}
& \left\|\left(D_{x_{n}}^{2}+R\right) \operatorname{op}\left(\Lambda^{-\frac{1}{2}}\right) u\right\|^{2}=\left\|D_{x_{n}}^{2} \operatorname{op}\left(\Lambda^{-\frac{1}{2}}\right) u\right\|^{2}+\left\|R \operatorname{op}\left(\Lambda^{-\frac{1}{2}}\right) u\right\|^{2} \\
& \quad+2 \operatorname{Re}\left(\mathrm{i}\left(D_{x_{n}} u, \operatorname{Rop}\left(\Lambda^{-1}\right) u\right)_{0}+\mathrm{i}\left(D_{x_{n}} u,\left[\operatorname{op}\left(\Lambda^{-\frac{1}{2}}\right), R\right] \operatorname{op}\left(\Lambda^{-\frac{1}{2}}\right) u\right)_{0}\right) \\
& \quad+2 \operatorname{Re}\left(\left(R D_{x_{n}} \operatorname{op}\left(\Lambda^{-\frac{1}{2}}\right) u, D_{x_{n}} \operatorname{op}\left(\Lambda^{-\frac{1}{2}}\right) u\right)+\left(D_{x_{n}} \operatorname{op}\left(\Lambda^{-\frac{1}{2}}\right) u,\left[D_{x_{n}}, R\right] \operatorname{op}\left(\Lambda^{-\frac{1}{2}}\right) u\right)\right) .
\end{aligned}
$$

Since, we have

$$
\left\|\operatorname{op}\left(\Lambda^{\frac{3}{2}}\right) u\right\|^{2}=\left(\operatorname{op}\left(\Lambda^{2}\right) \operatorname{op}\left(\Lambda^{\frac{1}{2}}\right) u, \operatorname{op}\left(\Lambda^{\frac{1}{2}}\right) u\right)=\sum_{j \leq n-1}\left(D_{j}^{2} \operatorname{op}\left(\Lambda^{\frac{1}{2}}\right) u, \operatorname{op}\left(\Lambda^{\frac{1}{2}}\right) u\right)+\tau^{2}\left(\operatorname{op}\left(\Lambda^{\frac{1}{2}}\right) u, \operatorname{op}\left(\Lambda^{\frac{1}{2}}\right) u\right) .
$$

By integration by parts, we find

$$
\left\|\mathrm{op}\left(\Lambda^{\frac{3}{2}}\right) u\right\|^{2}=\sum_{j \leq n-1}\left(D_{j} \mathrm{op}\left(\Lambda^{\frac{1}{2}}\right) u, D_{j} \mathrm{op}\left(\Lambda^{\frac{1}{2}}\right) u\right)+\tau^{2}\left\|\mathrm{op}\left(\Lambda^{\frac{1}{2}}\right) u\right\|^{2}=k+\tau^{2}\left\|\mathrm{op}\left(\Lambda^{\frac{1}{2}}\right) u\right\|^{2} .
$$

Let $\chi_{0} \in C_{0}^{\infty}\left(\mathbb{R}^{n+1}\right)$ such that $\chi_{0}=1$ in the support of $u$. We have

$$
k=\sum_{j \leq n-1}\left(\chi_{0} D_{j} \mathrm{op}\left(\Lambda^{\frac{1}{2}}\right) u, D_{j} \mathrm{op}\left(\Lambda^{\frac{1}{2}}\right) u\right)+\sum_{j \leq n-1}\left(\left(1-\chi_{0}\right) D_{j} \mathrm{op}\left(\Lambda^{\frac{1}{2}}\right) u, D_{j} \mathrm{op}\left(\Lambda^{\frac{1}{2}}\right) u\right) .
$$

Recalling that $\chi_{0} u=u$, we obtain

$$
k=\sum_{j \leq n-1}\left(\chi_{0} D_{j} \mathrm{op}\left(\Lambda^{\frac{1}{2}}\right) u, D_{j} \mathrm{op}\left(\Lambda^{\frac{1}{2}}\right) u\right)+\sum_{j \leq n-1}\left(\left[\left(1-\chi_{0}\right), D_{j} \mathrm{op}\left(\Lambda^{\frac{1}{2}}\right)\right] u, D_{j} \mathrm{op}\left(\Lambda^{\frac{1}{2}}\right) u\right)=k^{\prime}+k^{\prime \prime} .
$$

Using the fact that $\left[\left(1-\chi_{0}\right), D_{j} \mathrm{op}\left(\Lambda^{\frac{1}{2}}\right)\right] \in \mathcal{T} \mathcal{O}^{\frac{1}{2}}$ and $D_{j} \mathrm{op}\left(\Lambda^{\frac{1}{2}}\right) \in \mathcal{T} \mathcal{O}^{\frac{3}{2}}$, we show

$$
k^{\prime \prime} \leq C\|\operatorname{op}(\Lambda) u\|^{2} .
$$

Using the fact that $\sum_{j, k \leq n-1} \chi_{0} a_{j, k} D_{j} v \overline{D_{k} v} \geq \delta \chi_{0} \sum_{j \leq n-1}\left|D_{j} v\right|^{2}, \delta>0$, we obtain

$$
\begin{aligned}
k^{\prime} & \leq C \sum_{j, k \leq n-1}\left(\chi_{0} a_{j k} D_{j} \mathrm{op}\left(\Lambda^{\frac{1}{2}}\right) u, D_{k} \mathrm{op}\left(\Lambda^{\frac{1}{2}}\right) u\right) \\
& \leq C \sum_{j, k \leq n-1}\left(\left[\chi_{0}, a_{j k} D_{j} \mathrm{op}\left(\Lambda^{\frac{1}{2}}\right)\right] u, D_{k} \mathrm{op}\left(\Lambda^{\frac{1}{2}}\right) u\right)+\sum_{j, k \leq n-1}\left(a_{j k} D_{j} \mathrm{op}\left(\Lambda^{\frac{1}{2}}\right) u, D_{k} \mathrm{op}\left(\Lambda^{\frac{1}{2}}\right) u\right) .
\end{aligned}
$$

Using the fact that $\left[\chi_{0}, a_{j k} D_{j} \mathrm{op}\left(\Lambda^{\frac{1}{2}}\right)\right] \in \mathcal{T} \mathcal{O}^{\frac{1}{2}}$ and $D_{k} \mathrm{op}\left(\Lambda^{\frac{1}{2}}\right) u \in \mathcal{T} \mathcal{O}^{\frac{3}{2}}$, we obtain

$$
k^{\prime} \leq C\left(\sum_{j, k \leq n-1}\left(a_{j k} D_{j} \mathrm{op}\left(\Lambda^{\frac{1}{2}}\right) u, D_{k} \mathrm{op}\left(\Lambda^{\frac{1}{2}}\right) u\right)+\|\mathrm{op}(\Lambda) u\|^{2}\right) .
$$


By integration by parts and recalling that $R=\sum_{j, k \leq n-1} a_{j, k} D_{j} D_{k}$, we have

$$
\sum_{j, k \leq n-1}\left(a_{j k} D_{j} \mathrm{op}\left(\Lambda^{\frac{1}{2}}\right) u, D_{k} \mathrm{op}\left(\Lambda^{\frac{1}{2}}\right) u\right)=\left(R \mathrm{op}\left(\Lambda^{\frac{1}{2}}\right) u, \mathrm{op}\left(\Lambda^{\frac{1}{2}}\right) u+\sum_{j, k \leq n-1}\left(\left[D_{k}, a_{j k}\right] D_{j} \mathrm{op}\left(\Lambda^{\frac{1}{2}}\right) u, \mathrm{op}\left(\Lambda^{\frac{1}{2}}\right) u\right) .\right.
$$

Since $\left[D_{k}, a_{j k}\right] D_{j} \mathrm{op}\left(\Lambda^{\frac{1}{2}}\right) \in \mathcal{T} \mathcal{O}^{\frac{3}{2}}$, then

$$
\sum_{j, k \leq n-1}\left(\left[D_{k}, a_{j k}\right] D_{j} \mathrm{op}\left(\Lambda^{\frac{1}{2}}\right) u, \mathrm{op}\left(\Lambda^{\frac{1}{2}}\right) u\right) \leq C\|\mathrm{op}(\Lambda) u\|^{2} .
$$

Following (3.36), we obtain

$$
\sum_{j, k \leq n-1}\left(a_{j k} D_{j} \mathrm{op}\left(\Lambda^{\frac{1}{2}}\right) u, D_{k} \mathrm{op}\left(\Lambda^{\frac{1}{2}}\right) u\right) \leq C\left(\left(R \mathrm{op}\left(\Lambda^{\frac{1}{2}}\right) u, \mathrm{op}\left(\Lambda^{\frac{1}{2}}\right) u\right)+\|\mathrm{op}(\Lambda) u\|^{2}\right) .
$$

Since

$$
\left(R \mathrm{op}\left(\Lambda^{\frac{1}{2}}\right) u, \mathrm{op}\left(\Lambda^{\frac{1}{2}}\right) u\right)=\left(R \mathrm{op}\left(\Lambda^{-\frac{1}{2}}\right) u, \mathrm{op}\left(\Lambda^{\frac{3}{2}}\right) u\right)+\left(\left[\operatorname{op}\left(\Lambda^{-1}\right), R\right] \operatorname{op}\left(\Lambda^{\frac{1}{2}}\right) u, \mathrm{op}\left(\Lambda^{\frac{3}{2}}\right) u\right) .
$$

Using the fact that $\left[\mathrm{op}\left(\Lambda^{-1}\right), R\right] \operatorname{op}\left(\Lambda^{\frac{1}{2}}\right) \in \mathcal{T} \mathcal{O}^{\frac{1}{2}}$ and the Cauchy Schwartz inequality, we obtain

$$
\left(R o p\left(\Lambda^{\frac{1}{2}}\right) u, \operatorname{op}\left(\Lambda^{\frac{1}{2}}\right) u\right) \leq \epsilon C\left\|\operatorname{op}\left(\Lambda^{\frac{3}{2}}\right) u\right\|^{2}+\frac{C}{\epsilon}\left\|R \operatorname{op}\left(\Lambda^{-\frac{1}{2}}\right) u\right\|^{2}+C\|\operatorname{op}(\Lambda) u\|^{2} .
$$

Combining (3.32)-(3.35), (3.37) and (3.38), we obtain

$$
\left\|\mathrm{op}\left(\Lambda^{\frac{3}{2}}\right) u\right\|^{2} \leq \epsilon C\left\|\mathrm{op}\left(\Lambda^{\frac{3}{2}}\right) u\right\|^{2}+\frac{C}{\epsilon}\left\|R \operatorname{op}\left(\Lambda^{-\frac{1}{2}}\right) u\right\|^{2}+C\|\mathrm{op}(\Lambda) u\|^{2} .
$$

For $\epsilon$ small enough, we obtain

$$
\left\|\operatorname{Rop}\left(\Lambda^{-\frac{1}{2}}\right) u\right\|^{2} \geq C\left(\left\|\operatorname{op}\left(\Lambda^{\frac{3}{2}}\right) u\right\|^{2}-\tau^{2}\left\|\operatorname{op}\left(\Lambda^{\frac{1}{2}}\right) u\right\|^{2}\right) .
$$

Using the same computations, we show

$$
\left(R D_{x_{n}} \mathrm{op}\left(\Lambda^{-\frac{1}{2}}\right) u, D_{x_{n}} \mathrm{op}\left(\Lambda^{-\frac{1}{2}}\right) u\right) \geq C\left(\left\|D_{x_{n}} \mathrm{op}\left(\Lambda^{\frac{1}{2}}\right) u\right\|^{2}-\tau\left\|D_{x_{n}} u\right\|^{2}\right) .
$$

Combining (3.31), (3.39) and (3.40), we obtain

$$
\begin{aligned}
\left\|\left(D_{x_{n}}^{2}+R\right) \operatorname{op}\left(\Lambda^{-\frac{1}{2}}\right) u\right\|^{2} & +\left|\left(D_{x_{n}} u, R \operatorname{op}\left(\Lambda^{-1}\right) u\right)_{0}\right|+\left|\left(D_{x_{n}} u,\left[\operatorname{op}\left(\Lambda^{-\frac{1}{2}}\right), R\right] \mathrm{op}\left(\Lambda^{-\frac{1}{2}}\right) u\right)_{0}\right| \\
& +\left|\left(D_{x_{n}} \mathrm{op}\left(\Lambda^{-\frac{1}{2}}\right) u,\left[D_{x_{n}}, R\right] \mathrm{op}\left(\Lambda^{-\frac{1}{2}}\right) u\right)\right|+\tau\|u\|_{1, \tau}^{2} \\
& \geq C\left(\left\|D_{x_{n}}^{2} \mathrm{op}\left(\Lambda^{-\frac{1}{2}}\right) u\right\|^{2}+\left\|D_{x_{n}} \mathrm{op}\left(\Lambda^{\frac{1}{2}}\right) u\right\|^{2}+\left\|\operatorname{op}\left(\Lambda^{\frac{3}{2}}\right) u\right\|^{2}\right) .
\end{aligned}
$$

Since

$$
\left|\left(D_{x_{n}} u, R \operatorname{oop}\left(\Lambda^{-1}\right) u\right)_{0}\right|+\left|\left(D_{x_{n}} u,\left[\operatorname{op}\left(\Lambda^{-\frac{1}{2}}\right), R\right] \operatorname{op}\left(\Lambda^{-\frac{1}{2}}\right) u\right)_{0}\right| \leq C\left(\left|D_{x_{n}} u\right|^{2}+|u|_{1}^{2}\right)=C|u|_{1,0, \tau}^{2}
$$


and

$$
\left|\left(D_{x_{n}} \mathrm{op}\left(\Lambda^{-\frac{1}{2}}\right) u,\left[D_{x_{n}}, R\right] \mathrm{op}\left(\Lambda^{-\frac{1}{2}}\right) u\right)\right| \leq C \tau\|u\|_{1, \tau}^{2} .
$$

From (3.30), (3.41)-(3.43) and (3.22), we obtain

$$
\left\|D_{x_{n}}^{2} \mathrm{op}\left(\Lambda^{-\frac{1}{2}}\right) u\right\|^{2}+\left\|D_{x_{n}} \mathrm{op}\left(\Lambda^{\frac{1}{2}}\right) u\right\|^{2}+\left\|\mathrm{op}\left(\Lambda^{\frac{3}{2}}\right) u\right\|^{2} \leq C\left(\|P(x, D, \tau, \mu) u\|^{2}+\tau|u|_{1,0, \tau}^{2}\right) .
$$

Following (3.23), we obtain (3.24).

We are now ready to prove that Theorem 3.2 and Lemma 3.2 imply Theorem 3.1.

Let $\chi \in C_{0}^{\infty}\left(\mathbb{R}^{n+1}\right)$ such that $\chi=1$ in the support of $w$ and $u=\chi \mathrm{op}\left(\Lambda^{-\frac{1}{2}}\right) w$. Then

$$
\begin{aligned}
P u= & \operatorname{op}\left(\Lambda^{-\frac{1}{2}}\right) P w+\left[P, \mathrm{op}\left(\Lambda^{-\frac{1}{2}}\right)\right] w+P\left[\chi, \mathrm{op}\left(\Lambda^{-\frac{1}{2}}\right)\right] w \\
= & \mathrm{op}\left(\Lambda^{-\frac{1}{2}}\right) P w+\left[P, \mathrm{op}\left(\Lambda^{-\frac{1}{2}}\right)\right] w+D_{x_{n}}^{2}\left[\chi, \mathrm{op}\left(\Lambda^{-\frac{1}{2}}\right)\right] w \\
& +R\left[\chi, \mathrm{op}\left(\Lambda^{-\frac{1}{2}}\right)\right] w+\tau c_{1}(x) D_{x_{n}}\left[\chi, \mathrm{op}\left(\Lambda^{-\frac{1}{2}}\right)\right] w \\
& +\tau T_{1}\left[\chi, \mathrm{op}\left(\Lambda^{-\frac{1}{2}}\right)\right] w+\tau^{2} C_{0}\left[\chi, \mathrm{op}\left(\Lambda^{-\frac{1}{2}}\right)\right] w \\
= & \mathrm{op}\left(\Lambda^{-\frac{1}{2}}\right) P w+\left[P, \mathrm{op}\left(\Lambda^{-\frac{1}{2}}\right)\right] w+a_{1}+a_{2}+a_{3}+a_{4}+a_{5} .
\end{aligned}
$$

Let us estimate $a_{1}, a_{2}, a_{3}, a_{4}$ and $a_{5}$. Recalling that $\left[\chi, \operatorname{op}\left(\Lambda^{-\frac{1}{2}}\right)\right] \in \mathcal{T} \mathcal{O}^{-\frac{3}{2}}$ and $\chi w=w$. Using the fact that $\left[D_{x_{n}}, T_{k}\right] \in \mathcal{T} \mathcal{O}^{k}$ for all $T_{k} \in \mathcal{T} \mathcal{O}^{k}$, we show

$$
\left\|a_{1}\right\|^{2} \leq C\left(\left\|D_{x_{n}}^{2} \mathrm{op}\left(\Lambda^{-\frac{3}{2}}\right) w\right\|^{2}+\left\|D_{x_{n}} \mathrm{op}\left(\Lambda^{-\frac{3}{2}}\right) w\right\|^{2}+\left\|\mathrm{op}\left(\Lambda^{-\frac{3}{2}}\right) w\right\|^{2}\right)
$$

and

$$
\left\|a_{3}\right\|^{2} \leq C\left(\tau^{2}\left\|D_{x_{n}} \mathrm{op}\left(\Lambda^{-\frac{3}{2}}\right) w\right\|^{2}+\tau^{2}\left\|\mathrm{op}\left(\Lambda^{-\frac{3}{2}}\right) w\right\|^{2}\right) .
$$

We have $R\left[\chi, \mathrm{op}\left(\Lambda^{-\frac{1}{2}}\right)\right] \in \mathcal{T} \mathcal{O}^{\frac{1}{2}}, T_{1}\left[\chi, \mathrm{op}\left(\Lambda^{-\frac{1}{2}}\right)\right] \in \mathcal{T} \mathcal{O}^{-\frac{1}{2}}$ and $C_{0}\left[\chi, \mathrm{op}\left(\Lambda^{-\frac{1}{2}}\right)\right] \in \mathcal{T} \mathcal{O}^{-\frac{3}{2}}$. Then we obtain

$$
\left\|a_{2}\right\|^{2}+\left\|a_{4}\right\|^{2}+\left\|a_{5}\right\|^{2} \leq C\left\|\operatorname{op}\left(\Lambda^{\frac{1}{2}}\right) w\right\|^{2} .
$$

Using the same computations made in the proof of Lemma 3.2 ( $c f . t_{1}, t_{2}$ and $t_{3}$ of (3.26)), we show

$$
\left\|\left[P, \operatorname{op}\left(\Lambda^{-\frac{1}{2}}\right)\right] w\right\|^{2} \leq C\left(\left\|\operatorname{op}\left(\Lambda^{\frac{1}{2}}\right) w\right\|^{2}+\tau^{-1}\left\|D_{x_{n}} w\right\|^{2}\right) .
$$

Following (3.44)-(3.48), we obtain

$$
\|P u\|^{2} \leq C\left(\tau^{-1}\|P w\|^{2}+\left\|\mathrm{op}\left(\Lambda^{\frac{1}{2}}\right) w\right\|^{2}+\tau^{-1}\left\|D_{x_{n}} w\right\|^{2}+\mu^{-1}\left\|D_{x_{n}}^{2} \mathrm{op}\left(\Lambda^{-1}\right) w\right\|^{2}\right) .
$$

We have

$$
\operatorname{op}\left(b_{1}\right) u=\operatorname{op}\left(b_{1}\right) \chi \mathrm{op}\left(\Lambda^{-\frac{1}{2}}\right) w=\operatorname{op}\left(\Lambda^{-\frac{1}{2}}\right) \operatorname{op}\left(b_{1}\right) w+\mathrm{op}\left(b_{1}\right)\left[\chi, \mathrm{op}\left(\Lambda^{-\frac{1}{2}}\right)\right] w+\left[\operatorname{op}\left(b_{1}\right), \mathrm{op}\left(\Lambda^{-\frac{1}{2}}\right)\right] w .
$$

Recalling that $\mathrm{op}\left(b_{1}\right) \in \mathcal{T} \mathcal{O}^{1}$, we obtain

$$
\frac{\tau}{\mu^{2}}\left|\operatorname{op}\left(b_{1}\right) u\right|_{1}^{2}=\frac{\tau}{\mu^{2}}\left|\operatorname{op}(\Lambda) \operatorname{op}\left(b_{1}\right) u\right|^{2} \leq C\left(\frac{\tau}{\mu^{2}}\left|\operatorname{op}\left(\Lambda^{\frac{1}{2}}\right) \operatorname{op}\left(b_{1}\right) w\right|^{2}+\frac{\tau}{\mu^{2}}\left|\operatorname{op}\left(\Lambda^{\frac{1}{2}}\right) w\right|^{2}\right) .
$$


We have

$$
\operatorname{op}\left(b_{2}\right) u=\operatorname{op}\left(b_{2}\right) \chi \mathrm{op}\left(\Lambda^{-\frac{1}{2}}\right) w=\operatorname{op}\left(\Lambda^{-\frac{1}{2}}\right) \operatorname{op}\left(b_{2}\right) w+\operatorname{op}\left(b_{2}\right)\left[\chi, \mathrm{op}\left(\Lambda^{-\frac{1}{2}}\right)\right] w+\left[\operatorname{op}\left(b_{2}\right), \operatorname{op}\left(\Lambda^{-\frac{1}{2}}\right)\right] w .
$$

Recalling that $\operatorname{op}\left(b_{2}\right) \in D_{x_{n}}+\mathcal{T} \mathcal{O}^{1}$, we obtain

$$
\tau\left|\operatorname{op}\left(b_{2}\right) u\right|^{2} \leq C\left(\tau\left|\operatorname{op}\left(\Lambda^{-\frac{1}{2}}\right) \operatorname{op}\left(b_{2}\right) w\right|^{2}+\tau\left|\operatorname{op}\left(\Lambda^{-\frac{1}{2}}\right) w\right|^{2}+\tau\left|D_{x_{n}} \mathrm{op}\left(\Lambda^{-\frac{3}{2}}\right) w\right|^{2}\right)
$$

Moreover, we have

$$
\tau|u|_{1,0, \tau}^{2}=\tau|u|_{1}^{2}+\tau\left|D_{x_{n}} u\right|^{2}=\tau|\operatorname{op}(\Lambda) u|^{2}+\tau\left|D_{x_{n}} u\right|^{2}
$$

We can write

$$
\operatorname{op}(\Lambda) u=\operatorname{op}(\Lambda) \chi \mathrm{op}\left(\Lambda^{-\frac{1}{2}}\right) w=\operatorname{op}\left(\Lambda^{\frac{1}{2}}\right) w+\operatorname{op}(\Lambda)\left[\chi, \mathrm{op}\left(\Lambda^{-\frac{1}{2}}\right)\right] w
$$

Then

$$
\tau|\operatorname{op}(\Lambda) u|^{2} \geq \tau\left|\operatorname{op}\left(\Lambda^{\frac{1}{2}}\right) w\right|^{2}-C \tau\left|\operatorname{op}\left(\Lambda^{-\frac{1}{2}}\right) w\right|^{2} \geq \tau\left|\operatorname{op}\left(\Lambda^{\frac{1}{2}}\right) w\right|^{2}-C \tau^{-1}\left|\operatorname{op}\left(\Lambda^{\frac{1}{2}}\right) w\right|^{2}
$$

For $\tau$ large enough, we obtain

$$
\tau|\operatorname{op}(\Lambda) u|^{2} \geq C \tau\left|\operatorname{op}\left(\Lambda^{\frac{1}{2}}\right) w\right|^{2}
$$

By the same way, we prove, for $\tau$ large enough

$$
\tau\left|D_{x_{n}} u\right|^{2} \geq C \tau \mid D_{x_{n}} \text { op }\left.\left(\Lambda^{-\frac{1}{2}}\right) w\right|^{2}
$$

Combining (3.52) and (3.53), we obtain

$$
\tau|u|_{1,0, \tau}^{2} \geq C\left(\tau\left|\operatorname{op}\left(\Lambda^{\frac{1}{2}}\right) w\right|^{2}+\tau\left|D_{x_{n}} \mathrm{op}\left(\Lambda^{-\frac{1}{2}}\right) w\right|^{2}\right)
$$

By the same way, we prove

$$
\begin{gathered}
\left\|\operatorname{op}\left(\Lambda^{\frac{3}{2}}\right) u\right\|^{2} \geq\|\operatorname{op}(\Lambda) w\|^{2}-C\|w\|^{2} \\
\left\|D_{x_{n}} \mathrm{op}\left(\Lambda^{\frac{1}{2}}\right) u\right\|^{2} \geq\left\|D_{x_{n}} w\right\|^{2}-C\left\|\operatorname{op}\left(\Lambda^{-1}\right) D_{x_{n}} w\right\|^{2}-C\left\|\operatorname{op}\left(\Lambda^{-1}\right) w\right\|^{2}
\end{gathered}
$$

and

$$
\left\|D_{x_{n}}^{2} \mathrm{op}\left(\Lambda^{-\frac{1}{2}}\right) u\right\|^{2} \geq\left\|D_{x_{n}}^{2} \mathrm{op}\left(\Lambda^{-1}\right) w\right\|^{2}-C\left\|D_{x_{n}}^{2} \mathrm{op}\left(\Lambda^{-2}\right) w\right\|^{2}-C\left\|D_{x_{n}} \mathrm{op}\left(\Lambda^{-2}\right) w\right\|^{2}-C\left\|\operatorname{op}\left(\Lambda^{-2}\right) w\right\|^{2}
$$

Combining (3.55)-(3.57), we obtain for $\tau$ large enough

$$
\left\|D_{x_{n}}^{2} \operatorname{op}\left(\Lambda^{-\frac{1}{2}}\right) u\right\|^{2}+\left\|D_{x_{n}} \mathrm{op}\left(\Lambda^{\frac{1}{2}}\right) u\right\|^{2}+\left\|\operatorname{op}\left(\Lambda^{\frac{3}{2}}\right) u\right\|^{2} \geq C\left(\left\|D_{x_{n}}^{2} \mathrm{op}\left(\Lambda^{-1}\right) w\right\|^{2}+\left\|D_{x_{n}} w\right\|^{2}+\|\operatorname{op}(\Lambda) w\|^{2}\right) .
$$

Combining (3.24), (3.49)-(3.51), (3.54) and (3.58), we obtain (3.10), for $\tau$ large enough and $|\mu| \geq \mu_{0}$.

\section{Proof of Theorem 3.2}

In this section, we use especially microlocal analysis and we recall and follow the notations used in [11]. The techniques used are the Calderon projector for the elliptic regions and Carleman estimates for non elliptic regions. 


\subsection{Study of the eigenvalues}

The proof is based on a partition argument related to the nature of the roots of the polynomial $p_{j}\left(x, \xi^{\prime}, \xi_{n}, \tau, \mu\right)$, $j=1,2$, in $\xi_{n}$. On $x_{n}=0$, we note

$$
q_{1}\left(x^{\prime}, \xi^{\prime}, \tau\right)=q_{1,1}\left(0, x^{\prime}, \xi^{\prime}, \tau\right)=q_{1,2}\left(0, x^{\prime}, \xi^{\prime}, \tau\right)
$$

Let us introduce the following microlocal regions

$$
\begin{aligned}
& \mathcal{E}_{1}^{+}=\left\{\left(x, \xi^{\prime}, \tau, \mu\right) \in K \times \mathbb{R}^{n-1} \times \mathbb{R} \times \mathbb{R}, \quad q_{2,1}+\frac{q_{1}^{2}}{\left(\frac{\partial \varphi_{1}}{\partial x_{n}}\right)^{2}}>0\right\}, \\
& \mathcal{Z}_{1}=\left\{\left(x, \xi^{\prime}, \tau, \mu\right) \in K \times \mathbb{R}^{n-1} \times \mathbb{R} \times \mathbb{R}, \quad q_{2,1}+\frac{q_{1}^{2}}{\left(\frac{\partial \varphi_{1}}{\partial x_{n}}\right)^{2}}=0\right\}, \\
& \mathcal{E}_{1}^{-}=\left\{\left(x, \xi^{\prime}, \tau, \mu\right) \in K \times \mathbb{R}^{n-1} \times \mathbb{R} \times \mathbb{R}, \quad q_{2,1}+\frac{q_{1}^{2}}{\left(\frac{\partial \varphi_{1}}{\partial x_{n}}\right)^{2}}<0\right\}, \\
& \mathcal{E}_{2}^{+}=\left\{\left(x, \xi^{\prime}, \tau, \mu\right) \in K \times \mathbb{R}^{n-1} \times \mathbb{R} \times \mathbb{R}, \quad q_{2,2}-\mu^{2}+\frac{q_{1}^{2}}{\left(\frac{\partial \varphi_{2}}{\partial x_{n}}\right)^{2}}>0\right\} \text {, } \\
& \mathcal{Z}_{2}=\left\{\left(x, \xi^{\prime}, \tau, \mu\right) \in K \times \mathbb{R}^{n-1} \times \mathbb{R} \times \mathbb{R}, \quad q_{2,2}-\mu^{2}+\frac{q_{1}^{2}}{\left(\frac{\partial \varphi_{2}}{\partial x_{n}}\right)^{2}}=0\right\}, \\
& \mathcal{E}_{2}^{-}=\left\{\left(x, \xi^{\prime}, \tau, \mu\right) \in K \times \mathbb{R}^{n-1} \times \mathbb{R} \times \mathbb{R}, \quad q_{2,2}-\mu^{2}+\frac{q_{1}^{2}}{\left(\frac{\partial \varphi_{2}}{\partial x_{n}}\right)^{2}}<0\right\} .
\end{aligned}
$$

We consider $p_{1 / 2}(x, \xi, \tau, \mu)$ as a polynomial in $\xi_{n}$. Then we have the following lemma describing the root localization of $p_{1 / 2}$ (here and in the sequel the index $\frac{1}{2} 1 / 2$ means 1 or 2 ).

Lemma 4.1. We have the following:

(1) For $\left(x, \xi^{\prime}, \tau, \mu\right) \in \mathcal{E}_{1 / 2}^{+}$, the roots of $p_{1 / 2}$ denoted $z_{1 / 2}^{ \pm}$satisfy $\pm \operatorname{Im} z_{1 / 2}^{ \pm}>0$.

(2) For $\left(x, \xi^{\prime}, \tau, \mu\right) \in \mathcal{Z}_{1 / 2}$, one of the roots of $p_{1 / 2}$ is real.

(3) For $\left(x, \xi^{\prime}, \tau, \mu\right) \in \mathcal{E}_{1 / 2}^{-}$, the roots of $p_{1 / 2}$ are in the half- plane $\operatorname{Im} \xi_{n}>0$ if $\frac{\partial \varphi_{1 / 2}}{\partial x_{n}}<0$ (resp. in the half-plane $\operatorname{Im} \xi_{n}<0$ if $\left.\frac{\partial \varphi_{1 / 2}}{\partial x_{n}}>0\right)$.

Proof. Using (3.3) and (3.4), we can write

$$
\left\{\begin{array}{l}
p_{1}\left(x^{\prime}, \xi, \tau, \mu\right)=\left(\xi_{n}+\mathrm{i} \tau \frac{\partial \varphi_{1}}{\partial x_{n}}-\mathrm{i} \alpha_{1}\right)\left(\xi_{n}+\mathrm{i} \tau \frac{\partial \varphi_{1}}{\partial x_{n}}+\mathrm{i} \alpha_{1}\right) \\
p_{2}\left(x^{\prime}, \xi, \tau, \mu\right)=\left(\xi_{n}+\mathrm{i} \frac{\partial \varphi_{2}}{\partial x_{n}}-\mathrm{i} \alpha_{2}\right)\left(\xi_{n}+\mathrm{i} \tau \frac{\partial \varphi_{2}}{\partial x_{n}}+\mathrm{i} \alpha_{2}\right)
\end{array}\right.
$$

where $\alpha_{j} \in \mathbb{C}, j=1,2$, defined by

$$
\left\{\begin{array}{l}
\alpha_{1}^{2}\left(x^{\prime}, \xi^{\prime}, \tau, \mu\right)=\left(\tau \frac{\partial \varphi_{1}}{\partial x_{n}}\right)^{2}+q_{2,1}+2 \mathrm{i} \tau q_{1} \\
\alpha_{2}^{2}\left(x^{\prime}, \xi^{\prime}, \tau, \mu\right)=\left(\tau \frac{\partial \varphi_{2}}{\partial x_{n}}\right)^{2}-\mu^{2}+q_{2,2}+2 \mathrm{i} \tau q_{1}
\end{array}\right.
$$


We set

$$
z_{1 / 2}^{ \pm}=-\mathrm{i} \tau \frac{\partial \varphi_{1 / 2}}{\partial x_{n}} \pm \mathrm{i} \alpha_{1 / 2}
$$

the roots of $p_{1 / 2}$. The imaginary parts of the roots of $p_{1 / 2}$ are

$$
-\tau \frac{\partial \varphi_{1 / 2}}{\partial x_{n}}-\operatorname{Re} \alpha_{1 / 2}, \quad-\tau \frac{\partial \varphi_{1 / 2}}{\partial x_{n}}+\operatorname{Re} \alpha_{1 / 2} .
$$

The signs of the imaginary parts are opposite if $\left|\partial \varphi_{1 / 2} / \partial x_{n}\right|<\left|\operatorname{Re} \alpha_{1 / 2}\right|$, equal to the sign of $-\partial \varphi_{1 / 2} / \partial x_{n}$ if $\left|\partial \varphi_{1 / 2} / \partial x_{n}\right|>\left|\operatorname{Re} \alpha_{1 / 2}\right|$ and one of the imaginary parts is null if $\left|\partial \varphi_{1 / 2} / \partial x_{n}\right|=\left|\operatorname{Re} \alpha_{1 / 2}\right|$. However the lines $\operatorname{Re} z= \pm \tau \partial \varphi_{1 / 2} / \partial x_{n}$ change by the application $z \mapsto z^{\prime}=z^{2}$ into the parabolic curve $\operatorname{Re} z^{\prime}=\left|\tau \partial \varphi_{1 / 2} / \partial x_{n}\right|^{2}-$ $\left|\operatorname{Im} z^{\prime}\right|^{2} / 4\left(\tau \partial \varphi_{1 / 2} / \partial x_{n}\right)^{2}$. Thus we obtain the lemma by replacing $z^{\prime}$ by $\alpha_{1 / 2}^{2}$.

Lemma 4.2. If we assume that the function $\varphi$ satisfies the following condition

$$
\left(\frac{\partial \varphi_{1}}{\partial x_{n}}\right)^{2}-\left(\frac{\partial \varphi_{2}}{\partial x_{n}}\right)^{2}>1
$$

then the following estimate holds

$$
q_{2,2}-\mu^{2}+\frac{q_{1}^{2}}{\left(\partial \varphi_{2} / \partial x_{n}\right)^{2}}>q_{2,1}+\frac{q_{1}^{2}}{\left(\partial \varphi_{1} / \partial x_{n}\right)^{2}}
$$

Proof. Following (3.4), on $\left\{x_{n}=0\right\}$, we have

$$
q_{2,2}\left(x, \xi^{\prime}, \tau\right)-q_{2,1}\left(x, \xi^{\prime}, \tau\right)=\left(\tau \frac{\partial \varphi_{1}}{\partial x_{n}}\right)^{2}-\left(\tau \frac{\partial \varphi_{2}}{\partial x_{n}}\right)^{2} \cdots
$$

Using (4.4), we have (4.5).

Remark 4.1. The result of this lemma imply that $\mathcal{E}_{1}^{+} \subset \mathcal{E}_{2}^{+}$.

\subsection{Estimate in $\mathcal{E}_{1}^{+}$}

In this part we study the problem in the elliptic region $\mathcal{E}_{1}^{+}$. In this region we can inverse the operator and use the Calderon projectors. Let $\chi^{+}\left(x, \xi^{\prime}, \tau, \mu\right) \in \mathcal{T} S_{\tau}^{0}$ such that in the support of $\chi^{+}$we have $q_{2,1}+\frac{q_{1}^{2}}{\left(\partial \varphi_{1} / \partial x_{n}\right)^{2}} \geq$ $\delta>0$. Then we have the following estimate.

Proposition 4.1. There exist constants $C>0, \tau_{0}>0$ and $\mu_{0}>0$ such that for any $\tau \geq \tau_{0},|\mu| \geq \mu_{0}$ such that $\tau \geq C_{0}|\mu|, C_{0}>0$, we have

$$
\tau^{2}\left\|\mathrm{op}\left(\chi^{+}\right) u\right\|_{1, \tau}^{2} \leq C\left(\|P(x, D, \tau, \mu) u\|^{2}+\|u\|_{1, \tau}^{2}+\tau|u|_{1,0, \tau}^{2}\right)
$$

for any $u \in C_{0}^{\infty}\left(\left\{x_{n} \geq 0\right\} \cap W\right)$.

If we suppose moreover that $\varphi$ satisfies (4.4) then the following estimate holds

$$
\tau\left|\operatorname{op}\left(\chi^{+}\right) u\right|_{1,0, \tau}^{2} \leq C\left(\|P(x, D, \tau, \mu) u\|^{2}+\frac{\tau}{\mu^{2}}\left|\operatorname{op}\left(b_{1}\right) u\right|_{1}^{2}+\tau\left|\operatorname{op}\left(b_{2}\right) u\right|^{2}+\|u\|_{1, \tau}^{2}+\tau^{-2}|u|_{1,0, \tau}^{2}\right)
$$

for any $u \in C_{0}^{\infty}\left(\left\{x_{n} \geq 0\right\} \cap W\right)$ and $b_{j}, j=1,2$, defined in (3.6). 
Proof. Let $\tilde{u}=\mathrm{op}\left(\chi^{+}\right) u$. From (3.2), we get

$$
\begin{cases}P \tilde{u}=\tilde{f} & \text { in }\left\{x_{n}>0\right\} \cap W, \\ o p\left(b_{1}\right) \tilde{u}=\left.\tilde{u}_{0}\right|_{x_{n}=0}-\left.\mathrm{i} \mu \tilde{v}_{0}\right|_{x_{n}=0}=\tilde{e}_{1} & \text { on }\left\{x_{n}=0\right\} \cap W, \\ \mathrm{op}\left(b_{2}\right) \tilde{u}=\left.\left(D_{x_{n}}+\mathrm{i} \tau \frac{\partial \varphi_{1}}{\partial x_{n}}\right) \tilde{u}_{0}\right|_{x_{n}=0}+\left.\left(D_{x_{n}}+\mathrm{i} \tau \frac{\partial \varphi_{2}}{\partial x_{n}}\right) \tilde{v}_{0}\right|_{x_{n}=0}=\tilde{e}_{2} & \text { on }\left\{x_{n}=0\right\} \cap W,\end{cases}
$$

with $\tilde{f}=\mathrm{op}\left(\chi^{+}\right) f+\left[P, \mathrm{op}\left(\chi^{+}\right)\right] u$. Since $\left[P, \mathrm{op}\left(\chi^{+}\right)\right] \in\left(\mathcal{T} \mathcal{O}^{0}\right) D_{x_{n}}+\mathcal{T} \mathcal{O}^{1}$, we have

$$
\|\tilde{f}\|_{L^{2}}^{2} \leq C\left(\|P(x, D, \tau, \mu) u\|_{L^{2}}^{2}+\|u\|_{1, \tau}^{2}\right)
$$

and $\tilde{e}_{1}=\mathrm{op}\left(\chi^{+}\right) e_{1}$ satisfying

$$
\left|\tilde{e}_{1}\right|_{1}^{2} \leq C\left|e_{1}\right|_{1}^{2}
$$

and

$$
\tilde{e}_{2}=\left.\left[\left(D_{x_{n}}+\mathrm{i} \tau \frac{\partial \varphi_{1}}{\partial x_{n}}\right), \mathrm{op}\left(\chi^{+}\right)\right] u_{0}\right|_{x_{n}=0}+\left.\left[\left(D_{x_{n}}+\mathrm{i} \tau \frac{\partial \varphi_{2}}{\partial x_{n}}\right), \mathrm{op}\left(\chi^{+}\right)\right] v_{0}\right|_{x_{n}=0}+\mathrm{op}\left(\chi^{+}\right) e_{2} .
$$

Since $\left[D_{x_{n}}, \operatorname{op}\left(\chi^{+}\right)\right] \in \mathcal{T} \mathcal{O}^{0}$, we have

$$
\left|\tilde{e}_{2}\right|^{2} \leq C\left(|u|^{2}+\left|e_{2}\right|^{2}\right) .
$$

Let $\underline{\tilde{u}}$ the extension of $\tilde{u}$ by 0 in $x_{n}<0$. According to (3.3)-(3.5), we obtain, by noting $\partial \varphi / \partial x_{n}=\operatorname{diag}\left(\partial \varphi_{1} / \partial x_{n}\right.$, $\left.\partial \varphi_{2} / \partial x_{n}\right), \gamma_{j}(\tilde{u})={ }^{t}\left(\left.D_{x_{n}}^{j}\left(\tilde{u}_{0}\right)\right|_{x_{n}=0^{+}},\left.D_{x_{n}}^{j}\left(\tilde{v}_{0}\right)\right|_{x_{n}=0^{+}}\right), j=0,1$ and $\delta^{(j)}=\left(\mathrm{d} / \mathrm{d} x_{n}\right)^{j}\left(\delta_{x_{n}=0}\right)$,

$$
P \underline{\tilde{u}}=\underline{\tilde{f}}-\gamma_{0}(\tilde{u}) \otimes \delta^{\prime}+\frac{1}{\mathrm{i}}\left(\gamma_{1}(\tilde{u})+2 \mathrm{i} \tau \frac{\partial \varphi}{\partial x_{n}}\right) \otimes \delta
$$

Let $\chi(x, \xi, \tau, \mu) \in S_{\tau}^{0}$ be equal to 1 for sufficiently large $|\xi|+\tau$ and in a neighborhood of $\operatorname{supp}\left(\chi^{+}\right)$. We assume $p$ is elliptic in support of $\chi$. These conditions are compatible due to the choice made for $\operatorname{supp}\left(\chi^{+}\right)$and Remark 4.1. Let $m$ large enough chosen later, by ellipticity of $p$ on $\operatorname{supp}(\chi)$ there exists a parametric $E=\operatorname{op}(e)$ of $P$. We recall that $e \in S_{\tau}^{-2}$ and $e$ has the following form $e(x, \xi, \tau, \mu)=\sum_{j=0}^{m} e_{j}(x, \xi, \tau, \mu)$, where $e_{0}=\chi p^{-1}$ and $e_{j}=\operatorname{diag}\left(e_{j, 1}, e_{j, 2}\right) \in S_{\tau}^{-2-j}$ where $e_{j, 1}$ and $e_{j, 2}$ are rational functions with respect to $\xi_{n}$. Then we have

$$
E P=\mathrm{op}(\chi)+R_{m}, \quad R_{m} \in \mathcal{O}^{-m-1} .
$$

Following (4.13) and (4.14), we obtain

$$
\left\{\begin{array}{l}
\underline{\tilde{u}}=E \underline{\tilde{f}}+E\left[-h_{1} \otimes \delta^{\prime}+\frac{1}{\mathrm{i}} h_{0} \otimes \delta\right]+w_{1}, \\
h_{0}=\gamma_{1}(\tilde{u})+2 \mathrm{i} \tau \frac{\partial \varphi}{\partial x_{n}} \gamma_{0}(\tilde{u}), \quad h_{1}=\gamma_{0}(\tilde{u}), \\
w_{1}=(\operatorname{Id}-\operatorname{op}(\chi)) \underline{\tilde{u}}-R_{m} \underline{\tilde{u}} .
\end{array}\right.
$$

Using the fact that $\operatorname{supp}(1-\chi) \cap \operatorname{supp}\left(\chi^{+}\right)=\varnothing$ and the symbolic calculus (see Lem. 2.10 in [8]), we have $(\mathrm{Id}-\mathrm{op}(\chi)) \mathrm{op}\left(\chi^{+}\right) \in \mathcal{O}^{-m}$, we obtain

$$
\left\|w_{1}\right\|_{2, \tau}^{2} \leq C \tau^{-2}\|u\|_{L^{2}}^{2} .
$$


Now, let us look at the term $E\left[-h_{1} \otimes \delta^{\prime}+\frac{1}{\mathrm{i}} h_{0} \otimes \delta\right]$. For $x_{n}>0$, we get

$$
\left\{\begin{array}{l}
E\left[-h_{1} \otimes \delta^{\prime}+\frac{1}{\mathrm{i}} h_{0} \otimes \delta\right]=\hat{T}_{1} h_{1}+\hat{T}_{0} h_{0}, \\
\hat{T}_{j}(h)=\left(\frac{1}{2 \pi}\right)^{n-1} \int \mathrm{e}^{\mathrm{i}\left(x^{\prime}-y^{\prime}\right) \xi^{\prime}} \hat{t}_{j}\left(x, \xi^{\prime}, \tau, \mu\right) h\left(y^{\prime}\right) \mathrm{d} y^{\prime} \mathrm{d} \xi^{\prime}=\mathrm{op}\left(\hat{t}_{j}\right) h \\
\hat{t}_{j}=\frac{1}{2 \pi \mathrm{i}} \int_{\gamma} \mathrm{e}^{\mathrm{i} x_{n} \xi_{n}} e(x, \xi, \tau, \mu) \xi_{n}^{j} \mathrm{~d} \xi_{n}
\end{array}\right.
$$

where $\gamma$ is the union of the segment $\left\{\xi_{n} \in \mathbb{R},\left|\xi_{n}\right| \leq c_{0} \sqrt{\left|\xi^{\prime}\right|^{2}+\tau^{2}}\right\}$ and the half circle $\left\{\xi_{n} \in \mathbb{C},\left|\xi_{n}\right|=\right.$ $\left.c_{0} \sqrt{\left|\xi^{\prime}\right|^{2}+\tau^{2}}, \operatorname{Im} \xi_{n}>0\right\}$, where the constant $c_{0}$ is chosen large enough for the roots $z_{1}^{+}$and $z_{2}^{+}$to be enclosed by $\gamma$ (if $c_{0}$ is large enough, the change of contour $\mathbb{R} \longrightarrow \gamma$ is possible because the symbol $e(x, \xi, \tau, \mu)$ is a holomorphic function for large $\left.\left|\xi_{n}\right| ; \xi_{n} \in \mathbb{C}\right)$. In particular we have in $x_{n} \geq 0$

$$
\left|\partial_{x_{n}}^{k} \partial_{x^{\prime}}^{\alpha} \partial_{\xi^{\prime}}^{\beta} \hat{t}_{j}\right| \leq C_{\alpha, \beta, k}\left\langle\xi^{\prime}, \tau\right\rangle^{j-1-|\beta|+k}, \quad j=0,1 .
$$

We now choose $\chi_{1}\left(x, \xi^{\prime}, \tau, \mu\right) \in \mathcal{T} S_{\tau}^{0}$, satisfying the same requirement as $\chi^{+}$, equal to 1 in a neighborhood of $\operatorname{supp}\left(\chi^{+}\right)$and such that the symbol $\chi$ be equal to 1 in a neighborhood of $\operatorname{supp}\left(\chi_{1}\right)$. We set $t_{j}=\chi_{1} \hat{t}_{j}, j=0,1$. Then we obtain

$$
\underline{\tilde{u}}=E \underline{\tilde{f}}+\mathrm{op}\left(t_{0}\right) h_{0}+\mathrm{op}\left(t_{1}\right) h_{1}+w_{1}+w_{2}
$$

where $w_{2}=\operatorname{op}\left(\left(1-\chi_{1}\right) \hat{t}_{0}\right) h_{0}+\mathrm{op}\left(\left(1-\chi_{1}\right) \hat{t}_{1}\right) h_{1}$. By using the composition formula of tangential operator, estimate (4.17), the fact that $\operatorname{supp}\left(1-\chi_{1}\right) \cap \operatorname{supp}\left(\chi^{+}\right)=\varnothing$ and the following trace formula

$$
\left|\gamma_{0}(u)\right|_{j} \leq C \tau^{-\frac{1}{2}}\|u\|_{j+1, \tau}, \quad j \in \mathbb{N}
$$

we obtain

$$
\left\|w_{2}\right\|_{2, \tau}^{2} \leq C \tau^{-2}\left(\|u\|_{1, \tau}^{2}+|u|_{1,0, \tau}^{2}\right)
$$

Since $\chi=1$ in the support of $\chi_{1}$, we have $e(x, \xi, \tau, \mu)$ is meromorphic w.r.t. $\xi_{n}$ in the support of $\chi_{1}$. The roots $z_{1 / 2}^{+}$are in $\operatorname{Im} \xi_{n} \geq c_{1} \sqrt{\left|\xi^{\prime}\right|^{2}+\tau^{2}}\left(c_{1}>0\right)$. If $c_{1}$ is small enough we can choose fixed contours $\gamma_{1 / 2}$ in $\operatorname{Im} \xi_{n} \geq \frac{c_{1}}{2} \sqrt{\left|\xi^{\prime}\right|^{2}+\tau^{2}}$ and we can write

$$
t_{j}=\operatorname{diag}\left(t_{j, 1}, t_{j, 2}\right), \quad t_{j, 1 / 2}\left(x, \xi^{\prime}, \tau, \mu\right)=\chi_{1}\left(x, \xi^{\prime}, \tau, \mu\right) \frac{1}{2 \pi \mathrm{i}} \int_{\gamma_{1 / 2}} \mathrm{e}^{\mathrm{i} x_{n} \xi_{n}} \mathrm{e}_{1 / 2}(x, \xi, \tau, \mu) \xi_{n}^{j} \mathrm{~d} \xi_{n}, \quad j=0,1 .
$$

Then there exists $c_{2}>0$ such that in $x_{n} \geq 0$, we obtain

$$
\left|\partial_{x_{n}}^{k} \partial_{x^{\prime}}^{\alpha} \partial_{\xi^{\prime}}^{\beta} t_{j}\right| \leq C_{\alpha, \beta, k} \mathrm{e}^{-c_{2} x_{n}\left\langle\xi^{\prime}, \tau\right\rangle}\left\langle\xi^{\prime}, \tau\right\rangle^{j-1-|\beta|+k} .
$$

In particular, we have $\mathrm{e}^{c_{2} x_{n} \tau}\left(\partial_{x_{n}}^{k}\right) t_{j}$ bounded in $\mathcal{T} S_{\tau}^{j-1+k}$ uniformly w.r.t. $x_{n} \geq 0$. Then

$$
\left\|\partial_{x^{\prime}} \mathrm{op}\left(t_{j}\right) h_{j}\right\|_{L^{2}}^{2}+\left\|\mathrm{op}\left(t_{j}\right) h_{j}\right\|_{L^{2}}^{2} \leq C \int_{x_{n}>0} \mathrm{e}^{-2 c_{2} x_{n} \tau}\left|\mathrm{op}\left(\mathrm{e}^{c_{2} x_{n} \tau} t_{j}\right) h_{j}\right|_{1}^{2}\left(x_{n}\right) \mathrm{d} x_{n} \leq C \tau^{-1}\left|h_{j}\right|_{j}^{2}
$$

and

$$
\left\|\partial_{x_{n}} \operatorname{op}\left(t_{j}\right) h_{j}\right\|_{L^{2}}^{2} \leq C \int_{x_{n}>0} \mathrm{e}^{-2 c_{2} x_{n} \tau}\left|\mathrm{op}\left(\mathrm{e}^{c_{2} x_{n} \tau} \partial_{x_{n}} t_{j}\right) h_{j}\right|_{L^{2}}^{2}\left(x_{n}\right) \mathrm{d} x_{n} \leq C \tau^{-1}\left|h_{j}\right|_{j}^{2}
$$


Using the fact that $h_{0}=\gamma_{1}(\tilde{u})+2 \mathrm{i} \tau \frac{\partial \varphi}{\partial x_{n}} \gamma_{0}(\tilde{u})$ and $h_{1}=\gamma_{0}(\tilde{u})$, we obtain

$$
\left\|\mathrm{op}\left(t_{j}\right) h_{j}\right\|_{1, \tau}^{2} \leq C \tau^{-1}|u|_{1,0, \tau}^{2}
$$

From (4.18) and estimates (4.10), (4.16), (4.20) and (4.23), we obtain (4.7).

It remains to proof (4.8). We recall that, in $\operatorname{supp}\left(\chi_{1}\right)$, we have

$$
e_{0}=\operatorname{diag}\left(e_{0,1}, e_{0,2}\right)=\operatorname{diag}\left(\frac{1}{p_{1}}, \frac{1}{p_{2}}\right)=\operatorname{diag}\left(\frac{1}{\left(\xi_{n}-z_{1}^{+}\right)\left(\xi_{n}-z_{1}^{-}\right)}, \frac{1}{\left(\xi_{n}-z_{2}^{+}\right)\left(\xi_{n}-z_{2}^{-}\right)}\right)
$$

Using the residue formula, we obtain

$$
\mathrm{e}^{-\mathrm{i} x_{n} z_{1 / 2}^{+} t_{j, 1 / 2}}=\chi_{1} \frac{\left(z_{1 / 2}^{+}\right)^{j}}{z_{1 / 2}^{+}-z_{1 / 2}^{-}}+\lambda_{1 / 2}, \quad j=0,1, \quad \lambda_{1 / 2} \in \mathcal{T} S_{\tau}^{-2+j} .
$$

Taking the traces of (4.18), we obtain

$$
\gamma_{0}(\tilde{u})=\operatorname{op}(c) \gamma_{0}(\tilde{u})+\mathrm{op}(d) \gamma_{1}(\tilde{u})+w_{0}
$$

where $w_{0}=\gamma_{0}\left(E \underline{\tilde{f}}+w_{1}+w_{2}\right)$ satisfies, according to the trace formula (4.19), the estimates (4.10), (4.16) and (4.20), the following estimate

$$
\tau\left|w_{0}\right|_{1}^{2} \leq C\left(\|P(x, D, \tau, \mu) u\|^{2}+\|u\|_{1, \tau}^{2}+\tau^{-2}|u|_{1,0, \tau}^{2}\right) .
$$

Following (4.23), $c$ and $d$ are two tangential symbols of order respectively 0 and -1 given by

$$
\begin{aligned}
& c_{0}=\operatorname{diag}\left(c_{0,1}, c_{0,2}\right) \quad \text { with } \quad c_{0,1 / 2}=-\left(\chi_{1} \frac{z_{1 / 2}^{-}}{z_{1 / 2}^{+}-z_{1 / 2}^{-}}\right), \\
& d_{-1}=\operatorname{diag}\left(d_{-1,1}, d_{-1,2}\right) \quad \text { with } \quad d_{-1,1 / 2}=\left(\chi_{1} \frac{1}{z_{1 / 2}^{+}-z_{1 / 2}^{-}}\right) .
\end{aligned}
$$

Following (4.9), the transmission conditions give

$$
\left\{\begin{array}{l}
\gamma_{0}\left(\tilde{u}_{0}\right)-\mathrm{i} \mu \gamma_{0}\left(\tilde{v}_{0}\right)=\tilde{e}_{1} \\
\gamma_{1}\left(\tilde{u}_{0}\right)+\gamma_{1}\left(\tilde{v}_{0}\right)+\mathrm{i} \tau \frac{\partial \varphi_{1}}{\partial x_{n}} \gamma_{0}\left(\tilde{u}_{0}\right)+\mathrm{i} \tau \frac{\partial \varphi_{2}}{\partial x_{n}} \gamma_{0}\left(\tilde{v}_{0}\right)=\tilde{e}_{2}
\end{array}\right.
$$

We recall that $\tilde{u}=\left(\tilde{u}_{0}, \tilde{v}_{0}\right)$, combining (4.25) and (4.27) we show that

$$
\operatorname{op}(k)^{t}\left(\gamma_{0}\left(\tilde{u}_{0}\right), \gamma_{0}\left(\tilde{v}_{0}\right), \Lambda^{-1} \gamma_{1}\left(\tilde{u}_{0}\right), \Lambda^{-1} \gamma_{1}\left(\tilde{v}_{0}\right)\right)=w_{0}+\frac{1}{\mu} \text { op }\left(\begin{array}{l}
0 \\
0 \\
1 \\
0
\end{array}\right) \tilde{e}_{1}+\text { op }\left(\begin{array}{l}
0 \\
0 \\
0 \\
1
\end{array}\right) \Lambda^{-1} \tilde{e}_{2}
$$


where $k$ is a $4 \times 4$ matrix, with principal symbol defined by

$$
k_{0}+\frac{1}{\mu} r_{0}=\left(\begin{array}{cccc}
1-c_{0,1} & 0 & -\Lambda d_{-1,1} & 0 \\
0 & 1-c_{0,2} & 0 & -\Lambda d_{-1,2} \\
0 & -\mathrm{i} & 0 & 0 \\
\mathrm{i} \tau \Lambda^{-1} \frac{\partial \varphi_{1}}{\partial x_{n}} & \mathrm{i} \tau \Lambda^{-1} \frac{\partial \varphi_{2}}{\partial x_{n}} & 1 & 1
\end{array}\right)+\frac{1}{\mu} r_{0}
$$

where $r_{0}$ is a tangential symbol of order 0 .

We now choose $\chi_{2}\left(x, \xi^{\prime}, \tau, \mu\right) \in \mathcal{T} S_{\tau}^{0}$, satisfying the same requirement as $\chi^{+}$, equal to 1 in a neighborhood of $\operatorname{supp}\left(\chi^{+}\right)$and such that the symbol $\chi_{1}$ be equal to 1 in a neighborhood of $\operatorname{supp}\left(\chi_{2}\right)$. In $\operatorname{supp}\left(\chi_{2}\right)$, we obtain

$$
\left.k_{0}\right|_{\operatorname{supp}\left(\chi_{2}\right)}=\left(\begin{array}{cccc}
\frac{z_{1}^{+}}{z_{1}^{+}-z_{1}^{-}} & 0 & -\frac{\Lambda}{z_{1}^{+}-z_{1}^{-}} & 0 \\
0 & \frac{z_{2}^{+}}{z_{2}^{+}-z_{2}^{-}} & 0 & -\frac{\Lambda}{z_{2}^{+}-z_{2}^{-}} \\
0 & -\mathrm{i} & 0 & 0 \\
\mathrm{i} \tau \Lambda^{-1} \frac{\partial \varphi_{1}}{\partial x_{n}} & \mathrm{i} \tau \Lambda^{-1} \frac{\partial \varphi_{2}}{\partial x_{n}} & 1 & 1
\end{array}\right)
$$

Then, following (4.3),

$$
\left.\operatorname{det}\left(k_{0}\right)\right|_{\operatorname{supp}\left(\chi_{2}\right)}=-\left(z_{1}^{+}-z_{1}^{-}\right)^{-1}\left(z_{2}^{+}-z_{2}^{-}\right)^{-1} \Lambda \alpha_{1}
$$

To prove that there exists $c>0$ such that $\left|\operatorname{det}\left(k_{0}\right)\right| \operatorname{supp}\left(\chi_{2}\right) \mid \geq c$, by homogeneity it suffices to prove that $\left.\operatorname{det}\left(k_{0}\right)\right|_{\operatorname{supp}\left(\chi_{2}\right)} \neq 0$ if $\left|\xi^{\prime}\right|^{2}+\tau^{2}=1$.

If we suppose that $\left.\operatorname{det}\left(k_{0}\right)\right|_{\operatorname{supp}\left(\chi_{2}\right)}=0$, we obtain $\alpha_{1}=0$ and then $\alpha_{1}^{2}=0$.

Following (4.2), we obtain

$$
q_{1}=0 \quad \text { and } \quad\left(\tau \frac{\partial \varphi_{1}}{\partial x_{n}}\right)^{2}+q_{2,1}=0 .
$$

But in $\mathcal{E}_{1}^{+}$, this implies $q_{2,1}>0$, then (4.29) is impossible.

Therefore $\left.\operatorname{det}\left(k_{0}\right)\right|_{\operatorname{supp}\left(\chi_{2}\right)} \neq 0$. It follows that, for $\tau$ large enough, $k=k_{0}+\frac{1}{\mu} r_{0}$ is elliptic in $\operatorname{supp}\left(\chi_{2}\right)$. Then there exists $l \in \mathcal{T} S_{\tau}^{0}$, such that

$$
\operatorname{op}(l) \operatorname{op}(k)=\operatorname{op}\left(\chi_{2}\right)+\tilde{R}_{m},
$$

with $\tilde{R}_{m} \in \mathcal{T} \mathcal{O}^{-m-1}$, for $m$ large enough. This yields

$$
\begin{gathered}
{ }^{t}\left(\gamma_{0}\left(\tilde{u}_{0}\right), \gamma_{0}\left(\tilde{v}_{0}\right), \Lambda^{-1} \gamma_{1}\left(\tilde{u}_{0}\right), \Lambda^{-1} \gamma_{1}\left(\tilde{v}_{0}\right)\right)=\operatorname{op}(l) w_{0}+\frac{1}{\mu} \mathrm{op}(l) \text { op }\left(\begin{array}{l}
0 \\
0 \\
1 \\
0
\end{array}\right) \tilde{e}_{1}+\operatorname{op}(l) \text { op }\left(\begin{array}{c}
0 \\
0 \\
0 \\
1
\end{array}\right) \Lambda^{-1} \tilde{e}_{2} \\
+\left(\operatorname{op}\left(1-\chi_{2}\right)-\tilde{R}_{m}\right)^{t}\left(\gamma_{0}\left(\tilde{u}_{0}\right), \gamma_{0}\left(\tilde{v}_{0}\right), \Lambda^{-1} \gamma_{1}\left(\tilde{u}_{0}\right), \Lambda^{-1} \gamma_{1}\left(\tilde{v}_{0}\right)\right)
\end{gathered}
$$


Since $\operatorname{supp}\left(1-\chi_{2}\right) \cap \operatorname{supp}\left(\chi^{+}\right)=\varnothing$ and by using (4.26), we obtain

$$
\tau|\tilde{u}|_{1,0, \tau}^{2} \leq C\left(\frac{\tau}{\mu^{2}}\left|\tilde{e}_{1}\right|_{1}^{2}+\tau\left|\tilde{e}_{2}\right|^{2}+\|P(x, D, \tau, \mu) u\|_{L^{2}}^{2}+\|u\|_{1, \tau}^{2}+\tau^{-2}|u|_{1,0, \tau}^{2}\right) .
$$

From estimates (4.11) and (4.12) and the trace formula (4.19), we obtain (4.8).

\subsection{Estimate in $\mathcal{Z}_{1}$}

The aim of this part is to prove the estimate in the region $\mathcal{Z}_{1}$. In this region, if $\varphi$ satisfies (4.4), the symbol $p_{1}(x, \xi, \tau, \mu)$ admits a real root and $p_{2}(x, \xi, \tau, \mu)$ admits two roots $z_{2}^{ \pm}$satisfy $\pm \operatorname{Im}\left(z_{2}^{ \pm}\right)>0$. Let $\chi^{0}\left(x, \xi^{\prime}, \tau, \mu\right) \in \mathcal{T} \mathcal{S}_{\tau}^{0}$ equal to 1 in $\mathcal{Z}_{1}$ and such that in the support of $\chi^{0}$ we have $q_{2,2}-\mu^{2}+\frac{q_{1}^{2}}{\left(\partial \varphi_{2} / \partial x_{n}\right)^{2}} \geq \delta>0$. Then we have the following estimate.

Proposition 4.2. There exist constants $C>0, \tau_{0}>0$ and $\mu_{0}>0$ such that for any $\tau \geq \tau_{0},|\mu| \geq \mu_{0}$ such that $\tau \geq C_{0}|\mu|, C_{0}>0$, we have the following estimate

$$
\tau\left\|\operatorname{op}\left(\chi^{0}\right) u\right\|_{1, \tau}^{2} \leq C\left(\|P(x, D, \tau, \mu) u\|^{2}+\tau|u|_{1,0, \tau}^{2}+\|u\|_{1, \tau}^{2}\right),
$$

for any $u \in C_{0}^{\infty}\left(\left\{x_{n} \geq 0\right\} \cap W\right)$.

If we assume moreover that $\varphi$ satisfies (4.4) then we have

$$
\tau\left|\operatorname{op}\left(\chi^{0}\right) u\right|_{1,0, \tau}^{2} \leq C\left(\|P(x, D, \tau, \mu) u\|^{2}+\frac{\tau}{\mu^{2}}\left|\operatorname{op}\left(b_{1}\right) u\right|_{1}^{2}+\tau\left|\operatorname{op}\left(b_{2}\right) u\right|^{2}+\|u\|_{1, \tau}^{2}+\tau^{-2}|u|_{1,0, \tau}^{2}\right),
$$

for any $u \in C_{0}^{\infty}\left(\left\{x_{n} \geq 0\right\} \cap W\right)$ and $b_{j}, j=1,2$, defined in (3.6).

\subsubsection{Preliminaries}

Let $u \in C_{0}^{\infty}(K), \tilde{u}=\mathrm{op}\left(\chi^{0}\right) u$ and $P$ the differential operator with principal symbol given by

$$
p(x, \xi, \tau, \mu)=\operatorname{diag}\left(p_{1}, p_{2}\right)
$$

with $p_{1}$ and $p_{2}$ defined in (3.7). Then we have the following system

$$
\begin{cases}P \tilde{u}=\tilde{f} & \text { in }\left\{x_{n}>0\right\} \cap W, \\ B \tilde{u}=\tilde{e}=\left(\tilde{e}_{1}, \tilde{e}_{2}\right) & \text { on }\left\{x_{n}=0\right\} \cap W,\end{cases}
$$

where $\tilde{f}=\mathrm{op}\left(\chi^{0}\right) f+\left[P, \mathrm{op}\left(\chi^{0}\right)\right] u$. Since $\left[P, \mathrm{op}\left(\chi^{0}\right)\right] \in\left(\mathcal{T} \mathcal{O}^{0}\right) D_{x_{n}}+\mathcal{T} \mathcal{O}^{1}$, we have

$$
\|\tilde{f}\|_{L^{2}}^{2} \leq C\left(\|P(x, D, \tau, \mu) u\|_{L^{2}}^{2}+\|u\|_{1, \tau}^{2}\right),
$$

$B$ defined in (3.6) and $\tilde{e}_{1}=\mathrm{op}\left(\chi^{0}\right) e_{1}$ satisfying

$$
\left|\tilde{e}_{1}\right|_{1}^{2} \leq C\left|e_{1}\right|_{1}^{2}
$$

and

$$
\tilde{e}_{2}=\left.\left[\left(D_{x_{n}}+\mathrm{i} \tau \frac{\partial \varphi_{1}}{\partial x_{n}}\right), \mathrm{op}\left(\chi^{0}\right)\right] u_{0}\right|_{x_{n}=0}+\left.\left[\left(D_{x_{n}}+\mathrm{i} \tau \frac{\partial \varphi_{2}}{\partial x_{n}}\right), \mathrm{op}\left(\chi^{0}\right)\right] v_{0}\right|_{x_{n}=0}+\mathrm{op}\left(\chi^{0}\right) e_{2} .
$$


Since $\left[D_{x_{n}}, o p\left(\chi^{+}\right)\right] \in \mathcal{T} \mathcal{O}^{0}$, we have

$$
\left|\tilde{e}_{2}\right|^{2} \leq C\left(|u|^{2}+\left|e_{2}\right|^{2}\right)
$$

Let us reduce the problem (4.32) to a first order system. Put $v={ }^{t}\left(\left\langle D^{\prime}, \tau\right\rangle \tilde{u}, D_{x_{n}} \tilde{u}\right)$. Then we obtain the following system

$$
\left\{\begin{array}{cc}
D_{x_{n}} v-\operatorname{op}(\mathcal{P}) v=F \quad \text { in }\left\{x_{n}>0\right\} \cap W, \\
\operatorname{op}(\mathcal{B}) v=\left(\frac{1}{\mu} \Lambda \tilde{e}_{1}, \tilde{e}_{2}\right) \quad \text { on }\left\{x_{n}=0\right\} \cap W,
\end{array}\right.
$$

where $\mathcal{P}$ is a $4 \times 4$ matrix, with principal symbol defined by

$$
\mathcal{P}_{0}=\left(\begin{array}{cc}
0 & \Lambda \mathrm{Id}_{2} \\
\Lambda^{-1} l_{2} & -\mathrm{i} l_{1}
\end{array}\right), \quad l_{1}=\left(\begin{array}{cc}
q_{1,1} & 0 \\
0 & q_{1,2}
\end{array}\right), \quad l_{2}=\left(\begin{array}{cc}
q_{2,1} & 0 \\
0 & q_{2,2}-\mu^{2}
\end{array}\right)
$$

$\mathcal{B}$ is a tangential symbol of order 0 , with principal symbol given by

$$
\mathcal{B}_{0}+\frac{1}{\mu} r_{0}=\left(\begin{array}{cccc}
0 & -\mathrm{i} & 0 & 0 \\
\mathrm{i} \Lambda^{-1} \frac{\partial \varphi_{1}}{\partial x_{n}} & \mathrm{i} \tau \Lambda^{-1} \frac{\partial \varphi_{2}}{\partial x_{n}} & 1 & 1
\end{array}\right)+\frac{1}{\mu} r_{0}
$$

$\left(r_{0}\right.$ a tangential symbol of order 0$), F={ }^{t}(0, \tilde{f})$ and $\Lambda=\left\langle\xi^{\prime}, \tau\right\rangle=\left(\left|\xi^{\prime}\right|^{2}+\tau^{2}\right)^{\frac{1}{2}}$.

For a fixed $\left(x_{0}, \xi_{0}^{\prime}, \tau_{0}, \mu_{0}\right)$ in supp $\chi_{0}$, the generalized eigenvalues of the matrix $\mathcal{P}$ are the zeroes in $\xi_{n}$ of $p_{1}$ and $p_{2}$ i.e. $z_{1}^{ \pm}=-\mathrm{i} \tau \frac{\partial \varphi_{1}}{\partial x_{n}} \pm \mathrm{i} \alpha_{1}$ and $z_{2}^{ \pm}=-\mathrm{i} \tau \frac{\partial \varphi_{2}}{\partial x_{n}} \pm \mathrm{i} \alpha_{2}$ with $\pm \operatorname{Im}\left(z_{2}^{ \pm}\right)>0$ and $z_{1}^{+} \in \mathbb{R}$.

Let $s\left(x, \xi^{\prime}, \tau, \mu\right)=\left(s_{1}^{-}, s_{2}^{-}, s_{1}^{+}, s_{2}^{+}\right)$a basis of the generalized eigenspace of $\mathcal{P}\left(x_{0}, \xi_{0}^{\prime}, \tau_{0}, \mu_{0}\right)$ corresponding to eigenvalues with positive or negative imaginary parts. The vectors $s_{j}^{ \pm}\left(x, \xi^{\prime}, \tau, \mu\right), j=1,2$ are $C^{\infty}$ functions on a conic neighborhood of $\left(x_{0}, \xi_{0}^{\prime}, \tau_{0}, \mu_{0}\right)$ of degree zero in $\left(\xi^{\prime}, \tau, \mu\right)$. We denote op $(s)\left(x, D_{x^{\prime}}, \tau, \mu\right)$ the pseudodifferential operator associated to the principal symbol

$$
s\left(x, \xi^{\prime}, \tau, \mu\right)=\left(s_{1}^{-}\left(x, \xi^{\prime}, \tau, \mu\right), s_{2}^{-}\left(x, \xi^{\prime}, \tau, \mu\right), s_{1}^{+}\left(x, \xi^{\prime}, \tau, \mu\right), s_{2}^{+}\left(x, \xi^{\prime}, \tau, \mu\right)\right) .
$$

Let $\hat{\chi}\left(x, \xi^{\prime}, \tau, \mu\right) \in \mathcal{T} S_{\tau}^{0}$ equal to 1 in a conic neighborhood of $\left(x_{0}, \xi_{0}^{\prime}, \tau_{0}, \mu_{0}\right)$ and in a neighborhood of $\operatorname{supp}\left(\chi^{0}\right)$ and satisfies that in the support of $\hat{\chi}, s$ is elliptic. Then there exists $n \in \mathcal{T} S_{\tau}^{0}$, such that

$$
\operatorname{op}(s) \operatorname{op}(n)=\operatorname{op}(\hat{\chi})+\hat{R}_{m}
$$

with $\hat{R}_{m} \in \mathcal{T} \mathcal{O}^{-m-1}$, for $m$ large.

Let $V=\operatorname{op}(n) v$. Then we have the following system

$$
\begin{cases}D_{x_{n}} V=G V+A V+F_{1} & \text { in }\left\{x_{n}>0\right\} \cap W, \\ \operatorname{op}\left(\mathcal{B}_{1}\right) V=\left(\frac{1}{\mu} \Lambda \tilde{e}_{1}, \tilde{e}_{2}\right)+v_{1} & \text { on }\left\{x_{n}=0\right\} \cap W,\end{cases}
$$

where $G=\operatorname{op}(n) \operatorname{op}(\mathcal{P}) \operatorname{op}(s), A=\left[D_{x_{n}}, \mathrm{op}(n)\right] \operatorname{op}(s), F_{1}=\operatorname{op}(n) F+\operatorname{op}(n) \operatorname{op}(\mathcal{P})\left(\operatorname{op}(1-\hat{\chi})-\hat{R}_{m}\right) v+$ $\left[D_{x_{n}}, \operatorname{op}(n)\right]\left(\operatorname{op}(1-\hat{\chi})-\hat{R}_{m}\right) v, \operatorname{op}\left(\mathcal{B}_{1}\right)=\operatorname{op}(\mathcal{B}) \operatorname{op}(s)$ and $v_{1}=\operatorname{op}(\mathcal{B})\left(\operatorname{op}(\hat{\chi}-1)+\hat{R}_{m}\right) v$.

Using the fact that $\operatorname{supp}(1-\hat{\chi}) \cap \operatorname{supp}\left(\chi^{0}\right)=\varnothing, \hat{R}_{m} \in \mathcal{T O}^{-m-1}$, for $m$ large and estimate (4.33), we show

$$
\left\|F_{1}\right\|^{2} \leq C\left(\|P(x, D, \tau, \mu) u\|_{L^{2}}^{2}+\|u\|_{1, \tau}^{2}\right) .
$$

Using the fact that $\operatorname{supp}(1-\hat{\chi}) \cap \operatorname{supp}\left(\chi^{0}\right)=\varnothing, \hat{R}_{m} \in \mathcal{T} \mathcal{O}^{-m-1}$, for $m$ large and the trace formula (4.19), we show

$$
\tau\left|v_{1}\right|^{2} \leq C\left(\tau^{-2}|u|_{1,0, \tau}^{2}+\|u\|_{1, \tau}^{2}\right)
$$


Here we recall an argument shown in Taylor [14] given by this lemma:

Lemma 4.3. Let $v$ solves the system

$$
\frac{\partial}{\partial y} v=G v+A v
$$

where $G=\left(\begin{array}{cc}E & \\ & F\end{array}\right)$ and $A$ are pseudo-differential operators of order 1 and 0 , respectively. We suppose that the symbols of $E$ and $F$ are two square matrices and have disjoint sets of eigenvalues. Then there exists a pseudo-differential operator $K$ of order -1 such that $w=(I+K) v$ satisfies

$$
\frac{\partial}{\partial y} w=G w+\left(\begin{array}{cc}
\alpha_{1} & \\
& \alpha_{2}
\end{array}\right) w+R_{1} w+R_{2} v
$$

where $\alpha_{j}$ and $R_{j}, j=1,2$ are pseudo-differential operators of order 0 and $-\infty$, respectively.

By this argument, there exists a pseudo-differential operator $K\left(x, D_{x^{\prime}}, \tau, \mu\right)$ of order -1 such that the boundary problem (4.37) is reduced to the following

$$
\begin{cases}D_{x_{n}} w-\operatorname{op}(\mathcal{H}) w=\tilde{F} & \text { in }\left\{x_{n}>0\right\} \cap W \\ \operatorname{op}(\tilde{\mathcal{B}}) w=\left(\frac{1}{\mu} \Lambda \tilde{e}_{1}, \tilde{e}_{2}\right)+v_{1}+v_{2} & \text { on }\left\{x_{n}=0\right\} \cap W\end{cases}
$$

where $w=(I+K) V, \tilde{F}=(I+K) F_{1}, \mathrm{op}(\mathcal{H})$ is a tangential of order 1 with principal $\operatorname{symbol} \mathcal{H}^{\prime} \operatorname{diag}\left(\mathcal{H}^{-}, \mathcal{H}^{+}\right)$ and $-\operatorname{Im}\left(\mathcal{H}^{-}\right) \geq C \Lambda, \operatorname{op}(\tilde{\mathcal{B}})=\operatorname{op}\left(\mathcal{B}_{1}\right)\left(I+K^{\prime}\right)$ with $K^{\prime}$ is such that $\left(I+K^{\prime}\right)(I+K)=I d+R_{m}^{\prime}\left(R_{m}^{\prime} \in \mathcal{O}^{-m-1}\right.$, for $m$ large) and $v_{2}=\mathrm{op}\left(\mathcal{B}_{1}\right) R_{m}^{\prime} V$.

According to (4.38), we have

$$
\|\tilde{F}\|^{2} \leq C\left(\|P(x, D, \tau, \mu) u\|_{L^{2}}^{2}+\|u\|_{1, \tau}^{2}\right)
$$

Using the fact that $R_{m}^{\prime} \in \mathcal{O}^{-m-1}$, for $m$ large, the trace formula (4.19) and estimates (4.34), (4.35) and (4.39), we show

$$
\tau|\operatorname{op}(\tilde{\mathcal{B}}) w|^{2} \leq C\left(\frac{\tau}{\mu^{2}}\left|e_{1}\right|_{1}^{2}+\tau\left|e_{2}\right|^{2}+\tau^{-2}|u|_{1,0, \tau}^{2}+\|u\|_{1, \tau}^{2}\right) .
$$

Lemma 4.4. Let $\mathcal{R}=\operatorname{diag}\left(-\rho I d_{2}, 0\right), \rho>0$. Then there exists $C>0$ such that:

(1) $\operatorname{Im}(\mathcal{R H})=\operatorname{diag}\left(e\left(x, \xi^{\prime}, \tau, \mu\right), 0\right)$, with $e\left(x, \xi^{\prime}, \tau, \mu\right)=-\rho \operatorname{Im}\left(\mathcal{H}^{-}\right)$,

(2) $e\left(x, \xi^{\prime}, \tau, \mu\right) \geq C \Lambda$ in $\operatorname{supp}\left(\chi^{0}\right)$,

(3) $-\mathcal{R}+\tilde{\mathcal{B}}^{\star} \tilde{\mathcal{B}} \geq C$.Id on $\left\{x_{n}=0\right\} \cap W \cap \operatorname{supp}\left(\chi^{0}\right)$.

Proof. We have

$$
\operatorname{Im}(\mathcal{R H})=\operatorname{diag}\left(-\rho \operatorname{Im}\left(\mathcal{H}^{-}\right), 0\right)=\operatorname{diag}\left(e\left(x, \xi^{\prime}, \tau, \mu\right), 0\right),
$$

where $e\left(x, \xi^{\prime}, \tau, \mu\right)=-\rho \operatorname{Im}\left(\mathcal{H}^{-}\right) \geq C \Lambda, C>0$. It remains to prove $(3)$.

We denote the principal symbol $\tilde{\mathcal{B}}$ of the boundary operator op $(\tilde{\mathcal{B}})$ by $\left(\tilde{\mathcal{B}}^{-}, \tilde{\mathcal{B}}^{+}\right)$where $\tilde{\mathcal{B}}^{+}$is the restriction of $\tilde{\mathcal{B}}$ to subspace generated by $\left(s_{1}^{+}, s_{2}^{+}\right)$. We begin by proving that $\tilde{\mathcal{B}}^{+}$is an isomorphism. Denote

$$
w_{1}={ }^{t}(1,0) \text { and } w_{2}={ }^{t}(0,1)
$$

Then

$$
\left\{\begin{array}{l}
s_{1}^{+}=\left(w_{1}, z_{1}^{+} \Lambda^{-1} w_{1}\right) \\
s_{2}^{+}=\left(w_{2}, z_{2}^{+} \Lambda^{-1} w_{2}\right)
\end{array}\right.
$$


are eigenvectors associated to $z_{1}^{+}$and $z_{2}^{+}$. We have $\tilde{\mathcal{B}}^{+}=\left(\mathcal{B}_{0}+\frac{1}{\mu} r_{0}\right)\left(s_{1}^{+} s_{2}^{+}\right)=\mathcal{B}_{0}^{+}+\frac{1}{\mu} r_{0}^{+}$. To proof that $\tilde{\mathcal{B}}^{+}$is an isomorphism it suffices, for $\tau$ large, to proof that $\mathcal{B}_{0}^{+}$is an isomorphism. Following (4.3), we obtain

$$
\mathcal{B}_{0}^{+}=\left(\begin{array}{cc}
0 & -\mathrm{i} \\
\Lambda^{-1} \mathrm{i} \alpha_{1} & \Lambda^{-1} \mathrm{i} \alpha_{2}
\end{array}\right)
$$

Then

$$
\operatorname{det}\left(\mathcal{B}_{0}^{+}\right)=-\Lambda^{-1} \alpha_{1}
$$

If we suppose that $\operatorname{det}\left(\mathcal{B}_{0}^{+}\right)=0$, we obtain $\alpha_{1}=0$ and then $\alpha_{1}^{2}=0$. Following (4.2), we obtain

$$
q_{1}=0 \quad \text { and } \quad\left(\tau \frac{\partial \varphi_{1}}{\partial x_{n}}\right)^{2}+q_{2,1}=0 .
$$

Combining with the fact that $q_{2,1}+\frac{q_{1}^{2}}{\left(\partial \varphi_{1} / \partial x_{n}\right)^{2}}=0$, we obtain $\left(\tau \frac{\partial \varphi_{1}}{\partial x_{n}}\right)^{2}=0$, that is impossible because following (4.4), we have $\left(\frac{\partial \varphi_{1}}{\partial x_{n}}\right)^{2} \neq 0$ and following (3.4), we have $\tau \neq 0$. We deduce that $\tilde{\mathcal{B}}^{+}$is an isomorphism.

Let $w=\left(w^{-}, w^{+}\right) \in \mathbb{C}^{4}=\mathbb{C}^{2} \oplus \mathbb{C}^{2}$. Then we have $\tilde{\mathcal{B}} w=\tilde{\mathcal{B}}^{-} w^{-}+\tilde{\mathcal{B}}^{+} w^{+}$. Since $\tilde{\mathcal{B}}^{+}$is an isomorphism, then there exists a constant $C>0$ such that

$$
\left|\tilde{\mathcal{B}}^{+} w^{+}\right|^{2} \geq C\left|w^{+}\right|^{2}
$$

Therefore, we have

We deduce

$$
\left|w^{+}\right|^{2} \leq C\left(|\tilde{\mathcal{B}} w|^{2}+\left|w^{-}\right|^{2}\right)
$$

$$
-(\mathcal{R} w, w)=\rho\left|w^{-}\right|^{2} \geq \frac{1}{C}\left|w^{+}\right|^{2}+(\rho-1)\left|w^{-}\right|^{2}-|\tilde{\mathcal{B}} w|^{2} .
$$

Then, we obtain the result, if $\rho$ is large enough.

\subsubsection{Proof of Proposition 4.2}

We start by showing (4.30). We have

$$
\begin{aligned}
\left\|P_{1}(x, D, \tau, \mu) u_{0}\right\|^{2}= & \left\|\left(\operatorname{Re} P_{1}\right) u_{0}\right\|^{2}+\left\|\left(\operatorname{Im} P_{1}\right) u_{0}\right\|^{2} \\
& +\mathrm{i}\left[\left(\left(\operatorname{Im} P_{1}\right) u_{0},\left(\operatorname{Re} P_{1}\right) u_{0}\right)-\left(\left(\operatorname{Re} P_{1}\right) u_{0},\left(\operatorname{Im} P_{1}\right) u_{0}\right)\right] .
\end{aligned}
$$

By integration by parts we find

$$
\left\|P_{1}(x, D, \tau, \mu) u_{0}\right\|^{2}=\left\|\left(\operatorname{Re} P_{1}\right) u_{0}\right\|^{2}+\left\|\left(\operatorname{Im} P_{1}\right) u_{0}\right\|^{2}+\mathrm{i}\left(\left[\operatorname{Re} P_{1}, \operatorname{Im} P_{1}\right] u_{0}, u_{0}\right)+\tau Q_{0}\left(u_{0}\right),
$$

where

Then we have

$$
\left\{\begin{aligned}
Q_{0}\left(u_{0}\right)= & \left(-2 \frac{\partial \varphi_{1}}{\partial x_{n}} D_{x_{n}} u_{0}, D_{x_{n}} u_{0}\right)_{0}+\left(\mathrm{op}\left(r_{1}\right) u_{0}, D_{x_{n}} u_{0}\right)_{0} \\
& +\left(\operatorname{op}\left(r_{1}^{\prime}\right) D_{x_{n}} u_{0}, u_{0}\right)_{0}+\left(\mathrm{op}\left(r_{2}\right) u_{0}, u_{0}\right)_{0}+\tau\left(\frac{\partial \varphi_{1}}{\partial x_{n}} u_{0}, u_{0}\right)_{0} \\
& r_{1}=r_{1}^{\prime}=2 q_{1,1}, \quad r_{2}=-2 \frac{\partial \varphi_{1}}{\partial x_{n}} q_{2,1} .
\end{aligned}\right.
$$

$$
\left|Q_{0}\left(u_{0}\right)\right|^{2} \leq C\left|u_{0}\right|_{1,0, \tau}^{2}
$$


We obtain the same estimate on $v_{0}$ by the same method. In addition we know that the principal symbol of the operator $\left[\operatorname{Re} P_{j}, \operatorname{Im} P_{j}\right], j=1,2$, is given by $\frac{1}{\mathrm{i}}\left\{\operatorname{Re} P_{j}, \operatorname{Im} P_{j}\right\}$. Proceeding like Lebeau and Robbiano in paragraph 3 in [10], we obtain (4.30).

It remains to prove (4.31). Following Lemma 4.4 , let $G\left(x_{n}\right)=\mathrm{d} / \mathrm{d} x_{n}(\mathrm{op}(\mathcal{R}) w, w)_{L^{2}\left(\mathbb{R}^{n-1}\right)}$.

Using $D_{x_{n}} w-\mathrm{op}(\mathcal{H})=\tilde{F}$, we obtain

$$
G\left(x_{n}\right)=-2 \operatorname{Im}(\operatorname{op}(\mathcal{R}) \tilde{F}, w)-2 \operatorname{Im}(\operatorname{op}(\mathcal{R}) \operatorname{op}(\mathcal{H}) w, w) .
$$

The integration in the normal direction gives

$$
(\operatorname{op}(\mathcal{R}) w, w)_{0}=\int_{0}^{\infty} \operatorname{Im}(\operatorname{op}(\mathcal{R}) \operatorname{op}(\mathcal{H}) w, w) \mathrm{d} x_{n}+2 \int_{0}^{\infty} \operatorname{Im}(\operatorname{op}(\mathcal{R}) \tilde{F}, w) \mathrm{d} x_{n} .
$$

From Lemma 4.4 and the Gårding inequality, we obtain, for $\tau$ large enough,

$$
\operatorname{Im}(\operatorname{op}(\mathcal{R}) \operatorname{op}(\mathcal{H}) w, w) \geq C\left|w^{-}\right|_{\frac{1}{2}}^{2},
$$

moreover we have for all $\epsilon>0$

$$
\int_{0}^{\infty}|(\operatorname{op}(\mathcal{R}) \tilde{F}, w)| \mathrm{d} x_{n} \leq \epsilon C \tau\left\|w^{-}\right\|^{2}+\frac{C_{\epsilon}}{\tau}\|\tilde{F}\|^{2} .
$$

Applying Lemma 4.4 and the Gårding inequality, we obtain, for $\tau$ large enough,

$$
-(\operatorname{op}(\mathcal{R}) w, w)+|\operatorname{op}(\tilde{\mathcal{B}}) w|^{2} \geq C|w|^{2} .
$$

Combining (4.47), (4.46), (4.45) and (4.44), we get

$$
C\left|w^{-}\right|_{\frac{1}{2}}^{2}+C|w|^{2} \leq \frac{C}{\tau}\|\tilde{F}\|^{2}+|\operatorname{op}(\tilde{\mathcal{B}}) w|^{2} .
$$

Then

$$
\tau|w|^{2} \leq C\|\tilde{F}\|^{2}+\tau|\operatorname{op}(\tilde{\mathcal{B}}) w|^{2} .
$$

Recalling that $w=(I+K) V, V=\mathrm{op}(n) v, v={ }^{t}\left(\left\langle D^{\prime}, \tau\right\rangle \tilde{u}, D_{x_{n}} \tilde{u}\right)$ and $\tilde{u}=\mathrm{op}\left(\chi^{0}\right) u$ and using estimates (4.41) and (4.42), we prove (4.31).

\subsection{Estimate in $\mathcal{E}_{1}^{-}$}

Let $\chi^{-}\left(x, \xi^{\prime}, \tau, \mu\right) \in \mathcal{T} S_{\tau}^{0}$ equal to 1 in $\mathcal{E}_{1}^{-}$and such that in the support of $\chi^{-}$we have $q_{2,1}+\frac{q_{1}^{2}}{\left(\partial \varphi_{1} / \partial x_{n}\right)^{2}} \leq$ $-\delta<0$. Then we have the following estimate.

Proposition 4.3. There exist constants $C>0, \tau_{0}>0$ and $\mu_{0}>0$ such that for any $\tau \geq \tau_{0},|\mu| \geq \mu_{0}$ such that $\tau \geq C_{0}|\mu|, C_{0}>0$, we have the following estimate

$$
\tau\left\|\operatorname{op}\left(\chi^{-}\right) u\right\|_{1, \tau}^{2} \leq C\left(\|P(x, D, \tau, \mu) u\|^{2}+\tau|u|_{1,0, \tau}^{2}+\|u\|_{1, \tau}^{2}\right),
$$

for any $u \in C_{0}^{\infty}\left(\left\{x_{n} \geq 0\right\} \cap W\right)$.

Moreover if we assume $\frac{\partial \varphi_{1}}{\partial x_{n}}>0$, we have

$$
\tau\left|\operatorname{op}\left(\chi^{-}\right) u_{0}\right|_{1,0, \tau}^{2} \leq C\left(\|P(x, D, \tau, \mu) u\|^{2}+\tau^{-2}|u|_{1,0, \tau}^{2}+\|u\|_{1, \tau}^{2}\right)
$$

for any $u=\left(u_{0}, v_{0}\right) \in C_{0}^{\infty}\left(\left\{x_{n} \geq 0\right\} \cap W\right)$. 
Proof. Let $\tilde{u}=\mathrm{op}\left(\chi^{-}\right) u=\left(\mathrm{op}\left(\chi^{-}\right) u_{0}, \mathrm{op}\left(\chi^{-}\right) v_{0}\right)=\left(\tilde{u}_{0}, \tilde{v}_{0}\right)$.

In this region we have not a priori information for the roots of $p_{2}(x, \xi, \tau, \mu)$. Following the proof of (4.30), we obtain

$$
\tau\left\|\operatorname{op}\left(\chi^{-}\right) v_{0}\right\|_{1, \tau}^{2} \leq C\left(\left\|P(x, D, \tau, \mu) v_{0}\right\|^{2}+\tau\left|v_{0}\right|_{1,0, \tau}^{2}+\left\|v_{0}\right\|_{1, \tau}^{2}\right) .
$$

In $\operatorname{supp}\left(\chi^{-}\right)$the two roots $z_{1}^{ \pm}$of $p_{1}(x, \xi, \tau, \mu)$ are in the half-plane $\operatorname{Im} \xi_{n}<0$. Then we can use the Calderon projector. By the same way that the proof of (4.7) and using the fact that the operators $t_{0,1}$ and $t_{1,1}$ vanish in $x_{n}>0$ (because the roots are in $\operatorname{Im} \xi_{n}<0$, see (4.21)), the counterpart of (4.18) is then

$$
\tilde{u}_{0}=E \underline{\tilde{f}}_{1}+w_{1,1}+w_{2,1}, \quad \text { for } x_{n}>0
$$

where $w_{1,1}$ and $w_{2,1}$ satisfy (4.16) and (4.20) respectively.

We then obtain (see proof of (4.7))

$$
\tau^{2}\left\|\operatorname{op}\left(\chi^{-}\right) u_{0}\right\|_{1, \tau}^{2} \leq C\left(\left\|P_{1}(x, D, \tau, \mu) u_{0}\right\|^{2}+\tau\left|u_{0}\right|_{1,0, \tau}^{2}+\left\|u_{0}\right\|_{1, \tau}^{2}\right) .
$$

Combining (4.51) and (4.53), we obtain (4.49).

It remains to proof (4.50). We take the trace at $x_{n}=0^{+}$of $(4.52)$,

$$
\gamma_{0}\left(\tilde{u}_{0}\right)=w_{0,1}=\gamma_{0}\left(E \underline{\tilde{f}}_{1}+w_{1,1}+w_{2,1}\right),
$$

which, by the counterpart of (4.26), gives

$$
\tau\left|\gamma_{0}\left(\tilde{u}_{0}\right)\right|_{1}^{2} \leq C\left(\left\|P_{1}(x, D, \tau, \mu) u_{0}\right\|^{2}+\left\|u_{0}\right\|_{1, \tau}^{2}+\tau^{-2}\left|u_{0}\right|_{1,0, \tau}^{2}\right) .
$$

From (4.52) we also have

$$
D_{x_{n}} \tilde{u}_{0}=D_{x_{n}} E \underline{\tilde{f}}_{1}+D_{x_{n}} w_{1,1}+D_{x_{n}} w_{2,1}, \quad \text { for } x_{n}>0 .
$$

We take the trace at $x_{n}=0^{+}$and obtain

$$
\gamma_{1}\left(\tilde{u}_{0}\right)=\gamma_{0}\left(D_{x_{n}}\left(E \underline{\tilde{f}}_{1}+w_{1,1}+w_{2,1}\right)\right) .
$$

Using the trace formula (4.19), we obtain

$$
\left|\gamma_{1}\left(\tilde{u}_{0}\right)\right|^{2} \leq C \tau^{-1}\left\|D_{x_{n}}\left(E \underline{\tilde{f}}_{1}+w_{1,1}+w_{2,1}\right)\right\|_{1, \tau}^{2} \leq C \tau^{-1}\left\|E \underline{\tilde{f}}_{1}+w_{1,1}+w_{2,1}\right\|_{2, \tau}^{2}
$$

and, by the counterpart of (4.10), (4.16) and (4.20), this yields

$$
\tau\left|\gamma_{1}\left(\tilde{u}_{0}\right)\right|^{2} \leq C\left(\left\|P_{1}(x, D, \tau, \mu) u_{0}\right\|^{2}+\left\|u_{0}\right\|_{1, \tau}^{2}+\tau^{-2}\left|u_{0}\right|_{1,0, \tau}^{2}\right) .
$$

Combining (4.54) and (4.55), we obtain (4.50).

\subsection{End of the proof}

We can choose a partition of unity $\chi^{+}+\chi^{0}+\chi^{-}=1$ such that $\chi^{+}, \chi^{0}$ and $\chi^{-}$satisfy the properties listed in Propositions 4.1, 4.2 and 4.3 respectively. We have

$$
\|u\|_{1, \tau}^{2} \leq\left\|\mathrm{op}\left(\chi^{+}\right) u\right\|_{1, \tau}^{2}+\left\|\mathrm{op}\left(\chi^{0}\right) u\right\|_{1, \tau}^{2}+\left\|\mathrm{op}\left(\chi^{-}\right) u\right\|_{1, \tau}^{2} .
$$


Combining this inequality and (4.7), (4.30) and (4.49), we obtain, for $\tau$ large, the first estimate (3.22) of Theorem 3.2, i.e.

$$
\tau\|u\|_{1, \tau}^{2} \leq C\left(\|P(x, D, \tau, \mu) u\|^{2}+\tau|u|_{1,0, \tau}^{2}\right) .
$$

It remains to estimate $\tau|u|_{1,0, \tau}^{2}$. We begin by giving an estimate of $\tau\left|u_{0}\right|_{1,0, \tau}^{2}$.

We have

$$
\begin{gathered}
\left|u_{0}\right|_{1,0, \tau}^{2} \leq\left|\operatorname{op}\left(\chi^{+}\right) u_{0}\right|_{1,0, \tau}^{2}+\left|\operatorname{op}\left(\chi^{0}\right) u_{0}\right|_{1,0, \tau}^{2}+\left|\operatorname{op}\left(\chi^{-}\right) u_{0}\right|_{1,0, \tau}^{2}, \\
\left|\operatorname{op}\left(\chi^{+}\right) u_{0}\right|_{1,0, \tau}^{2} \leq\left|\operatorname{op}\left(\chi^{+}\right) u\right|_{1,0, \tau}^{2}
\end{gathered}
$$

and

$$
\left|\operatorname{op}\left(\chi^{0}\right) u_{0}\right|_{1,0, \tau}^{2} \leq\left|\mathrm{op}\left(\chi^{0}\right) u\right|_{1,0, \tau}^{2} .
$$

Combining these inequalities, (4.8), (4.31), (4.50) and the fact that $\tau^{-2}|u|_{1,0, \tau}^{2}=\tau^{-2}\left|u_{0}\right|_{1,0, \tau}^{2}+\tau^{-2}\left|v_{0}\right|_{1,0, \tau}^{2}$, we obtain, for $\tau$ large enough.

$$
\tau\left|u_{0}\right|_{1,0, \tau}^{2} \leq C\left(\|P(x, D, \tau, \mu) u\|^{2}+\frac{\tau}{\mu^{2}}\left|\operatorname{op}\left(b_{1}\right) u\right|_{1}^{2}+\tau\left|\operatorname{op}\left(b_{2}\right) u\right|^{2}+\tau^{-2}\left|v_{0}\right|_{1,0, \tau}^{2}+\|u\|_{1, \tau}^{2}\right) .
$$

For estimate $\tau\left|v_{0}\right|_{1,0, \tau}^{2}$, we use the transmission conditions given by (3.6). We have

$$
\mathrm{op}\left(b_{1}\right) u=\left.u_{0}\right|_{x_{n}=0}-\left.\mathrm{i} \mu v_{0}\right|_{x_{n}=0} \quad \text { on }\left\{x_{n}=0\right\} \cap W .
$$

Then

$$
\tau\left|v_{0}\right|_{1}^{2} \leq C\left(\frac{\tau}{\mu^{2}}\left|u_{0}\right|_{1}^{2}+\frac{\tau}{\mu^{2}}\left|\operatorname{op}\left(b_{1}\right) u\right|_{1}^{2}\right) .
$$

Since, for $|\mu| \geq \mu_{0}$, we have $\frac{\tau}{\mu^{2}}\left|u_{0}\right|_{1}^{2} \leq C \tau\left|u_{0}\right|_{1,0, \tau}^{2}$. Then using (4.56), we obtain

$$
\tau\left|v_{0}\right|_{1}^{2} \leq C\left(\|P(x, D, \tau, \mu) u\|^{2}+\frac{\tau}{\mu^{2}}\left|\operatorname{op}\left(b_{1}\right) u\right|_{1}^{2}+\tau\left|\operatorname{op}\left(b_{2}\right) u\right|^{2}+\tau^{-2}\left|v_{0}\right|_{1,0, \tau}^{2}+\|u\|_{1, \tau}^{2}\right) .
$$

We have also

$$
\operatorname{op}\left(b_{2}\right) u=\left.\left(D_{x_{n}}+\mathrm{i} \tau \frac{\partial \varphi_{1}}{\partial x_{n}}\right) u_{0}\right|_{x_{n}=0}+\left.\left(D_{x_{n}}+\mathrm{i} \tau \frac{\partial \varphi_{2}}{\partial x_{n}}\right) v_{0}\right|_{x_{n}=0} \quad \text { on }\left\{x_{n}=0\right\} \cap W .
$$

Then

$$
\tau\left|D_{x_{n}} v_{0}\right|^{2} \leq C\left(\tau\left|\operatorname{op}\left(b_{2}\right) u\right|^{2}+\tau\left|D_{x_{n}} u_{0}\right|^{2}+\tau^{3}\left|u_{0}\right|^{2}+\tau^{3}\left|v_{0}\right|^{2}\right) .
$$

Using the fact that $|u|_{k-1} \leq \tau^{-1}|u|_{k}$, we obtain

$$
\tau\left|D_{x_{n}} v_{0}\right|^{2} \leq C\left(\tau\left|\operatorname{op}\left(b_{2}\right) u\right|^{2}+\tau\left|D_{x_{n}} u_{0}\right|^{2}+\tau\left|u_{0}\right|_{1}^{2}+\tau\left|v_{0}\right|_{1}^{2}\right)
$$

Since we have $\tau\left|u_{0}\right|_{1,0, \tau}^{2}=\tau\left|D_{x_{n}} u_{0}\right|^{2}+\tau\left|u_{0}\right|_{1}^{2}$. Then using (4.56) and (4.57), we obtain

$$
\tau\left|D_{x_{n}} v_{0}\right|^{2} \leq C\left(\|P(x, D, \tau, \mu) u\|^{2}+\frac{\tau}{\mu^{2}}\left|\operatorname{op}\left(b_{1}\right) u\right|_{1}^{2}+\tau\left|\operatorname{op}\left(b_{2}\right) u\right|^{2}+\tau^{-2}\left|v_{0}\right|_{1,0, \tau}^{2}+\|u\|_{1, \tau}^{2}\right) .
$$

Combining (4.57) and (4.58), we have

$$
\tau\left|v_{0}\right|_{1,0, \tau}^{2} \leq C\left(\|P(x, D, \tau, \mu) u\|^{2}+\frac{\tau}{\mu^{2}}\left|\operatorname{op}\left(b_{1}\right) u\right|_{1}^{2}+\tau\left|\operatorname{op}\left(b_{2}\right) u\right|^{2}+\|u\|_{1, \tau}^{2}\right) .
$$


Combining (4.56) and (4.59), we obtain

$$
\tau|u|_{1,0, \tau}^{2} \leq C\left(\|P(x, D, \tau, \mu) u\|^{2}+\frac{\tau}{\mu^{2}}\left|\operatorname{op}\left(b_{1}\right) u\right|_{1}^{2}+\tau\left|\operatorname{op}\left(b_{2}\right) u\right|^{2}+\|u\|_{1, \tau}^{2}\right)
$$

Inserting (4.60) in (3.22) and for $\tau$ large enough, we obtain (3.23).

\section{Appendix A: Proof of Lemma 2.1}

To prove Lemma 2.1, we need to distinguish two cases.

(1) Inside $\mathcal{O}$

To simplify the expressions, we note $\|u\|_{L^{2}(\mathcal{O})}=\|u\|$.

Let $\chi \in C_{0}^{\infty}(\mathcal{O})$. We have by integration by part

$$
\left((\triangle-\mathrm{i}|\mu|) u, \chi^{2} u\right)=\left(-\nabla u, \chi^{2} \nabla u\right)-\left(\nabla u, \nabla\left(\chi^{2}\right) u\right)-\mathrm{i}|\mu|\|\chi u\|^{2} .
$$

Then

$$
|\mu|\|\chi u\|^{2} \leq C\left(\|f\|\left\|\chi^{2} u\right\|+\|\nabla u\|^{2}+\|\nabla u\|\|\chi u\|\right) .
$$

Then

$$
|\mu|\|\chi u\|^{2} \leq C\left(\frac{1}{\epsilon}\|f\|^{2}+\epsilon\left\|\chi^{2} u\right\|+\|\nabla u\|^{2}+\frac{1}{\epsilon}\|\nabla u\|^{2}+\epsilon\|\chi u\|^{2}\right) .
$$

Recalling that $|\mu| \geq \mu_{0}$, we have for $\epsilon$ small enough

$$
\|\chi u\|^{2} \leq C\left(\|\nabla u\|^{2}+\|f\|^{2}\right) .
$$

Hence the result inside $\mathcal{O}$.

(2) In the neighborhood of the boundary

Let $x=\left(x^{\prime}, x_{n}\right) \in \mathbb{R}^{n-1} \times \mathbb{R}$. Then

$$
\partial \mathcal{O}=\left\{x \in \mathbb{R}^{n}, x_{n}=0\right\}
$$

Let $\epsilon>0$ such that $0<x_{n}<\epsilon$. Then we have

$$
u\left(x^{\prime}, \epsilon\right)-u\left(x^{\prime}, x_{n}\right)=\int_{x_{n}}^{\epsilon} \partial_{x_{n}} u\left(x^{\prime}, \sigma\right) \mathrm{d} \sigma .
$$

Then

$$
\left|u\left(x^{\prime}, x_{n}\right)\right|^{2} \leq 2\left|u\left(x^{\prime}, \epsilon\right)\right|^{2}+2\left(\int_{x_{n}}^{\epsilon}\left|\partial_{x_{n}} u\left(x^{\prime}, \sigma\right)\right| \mathrm{d} \sigma\right)^{2} .
$$

Using the Cauchy Schwartz inequality, we obtain

$$
\left|u\left(x^{\prime}, x_{n}\right)\right|^{2} \leq 2\left|u\left(x^{\prime}, \epsilon\right)\right|^{2}+2 \epsilon^{2} \int_{0}^{\epsilon}\left|\partial_{x_{n}} u\left(x^{\prime}, x_{n}\right)\right|^{2} \mathrm{~d} x_{n} .
$$

Integrating with respect to $x^{\prime}$, we obtain

$$
\int_{\left|x^{\prime}\right|<\epsilon}\left|u\left(x^{\prime}, x_{n}\right)\right|^{2} \mathrm{~d} x^{\prime} \leq 2 \int_{\left|x^{\prime}\right|<\epsilon}\left|u\left(x^{\prime}, \epsilon\right)\right|^{2} \mathrm{~d} x^{\prime}+2 \epsilon^{2} \int_{\left|x^{\prime}\right|<\epsilon,\left|x_{n}\right|<\epsilon}\left(\left|\partial_{x_{n}} u\left(x^{\prime}, x_{n}\right)\right|^{2} \mathrm{~d} x_{n}\right) \mathrm{d} x^{\prime} .
$$


Using the trace theorem, we have

$$
\int_{\left|x^{\prime}\right|<\epsilon}\left|u\left(x^{\prime}, \epsilon\right)\right|^{2} \mathrm{~d} x^{\prime} \leq C \int_{\left|x^{\prime}\right|<2 \epsilon,\left|x_{n}-\epsilon\right|<\epsilon}\left(|u(x)|^{2}+|\nabla u(x)|^{2}\right) \mathrm{d} x .
$$

Now we introduce the following cut-off functions

$$
\chi_{1}(x)= \begin{cases}1 & \text { if } 0<x_{n}<\frac{\epsilon}{2}, \\ 0 & \text { if } x_{n}>\epsilon\end{cases}
$$

and

$$
\chi_{2}(x)= \begin{cases}1 & \text { if } \frac{\epsilon}{2}<x_{n}<\frac{3 \epsilon}{2}, \\ 0 & \text { if } x_{n}<\frac{\epsilon}{4}, x_{n}>2 \epsilon .\end{cases}
$$

Combining (4.62) and (4.63), we obtain for $\epsilon$ small enough

$$
\left\|\chi_{1} u\right\|^{2} \leq C\left(\left\|\chi_{2} u\right\|^{2}+\|\nabla u\|^{2}\right) .
$$

Since following (4.61), we have

$$
\left\|\chi_{2} u\right\|^{2} \leq C\left(\|f\|^{2}+\|\nabla u\|^{2}\right)
$$

Inserting in (4.64), we obtain

$$
\left\|\chi_{1} u\right\|^{2} \leq C\left(\|f\|^{2}+\|\nabla u\|^{2}\right) .
$$

Hence the result in the neighborhood of the boundary.

Following (4.61), we can write

$$
\left\|\left(1-\chi_{1}\right) u\right\|^{2} \leq C\left(\|f\|^{2}+\|\nabla u\|^{2}\right) .
$$

Adding (4.65) and (4.66), we obtain (2.9).

\section{Appendix B: Proof of Lemma 3.1}

Let $\chi \in C_{0}^{\infty}\left(\mathbb{R}^{n}\right)$ such that $\chi=1$ in the support of $u$. It suffices to show that $\mathrm{op}\left(\Lambda^{s}\right) \mathrm{e}^{\tau \varphi} \chi \mathrm{op}\left(\Lambda^{-s}\right)$ is bounded in $L^{2}$. Recalling that for all $u$ and $v \in \mathcal{S}\left(\mathbb{R}^{n}\right)$, we have

$$
\mathcal{F}(u v)\left(\xi^{\prime}\right)=\left(\frac{1}{2 \pi}\right)^{n-1} \mathcal{F}(u) * \mathcal{F}(v)\left(\xi^{\prime}\right), \quad \forall \xi^{\prime} \in \mathbb{R}^{n-1} .
$$

Then

$$
\begin{aligned}
\mathcal{F}\left(\mathrm{op}\left(\Lambda^{s}\right) \mathrm{e}^{\tau \varphi} \chi \mathrm{op}\left(\Lambda^{-s}\right) v\right)\left(\xi^{\prime}, \tau\right) & =\left\langle\xi^{\prime}, \tau\right\rangle^{s} \mathcal{F}\left(\mathrm{e}^{\tau \varphi} \chi \mathrm{op}\left(\Lambda^{-s}\right) v\right)\left(\xi^{\prime}, \tau\right) \\
& =\left(\frac{1}{2 \pi}\right)^{n-1}\left\langle\xi^{\prime}, \tau\right\rangle^{s}\left(g\left(\xi^{\prime}, \tau\right) *\left\langle\xi^{\prime}, \tau\right\rangle^{-s} \mathcal{F}(v)\right)\left(\xi^{\prime}, \tau\right),
\end{aligned}
$$


where $g\left(\xi^{\prime}, \tau\right)=\mathcal{F}\left(\mathrm{e}^{\tau \varphi} \chi\right)\left(\xi^{\prime}, \tau\right)$. Then we have

$$
\mathcal{F}\left(\operatorname{op}\left(\Lambda^{s}\right) \mathrm{e}^{\tau \varphi} \chi \mathrm{op}\left(\Lambda^{-s}\right) v\right)\left(\xi^{\prime}, \tau\right)=\int g\left(\xi^{\prime}-\eta^{\prime}, \tau\right)\left\langle\xi^{\prime}, \tau\right\rangle^{s}\left\langle\eta^{\prime}, \tau\right\rangle^{-s} \mathcal{F}(v)\left(\eta^{\prime}, \tau\right) \mathrm{d} \eta^{\prime}
$$

Let $k\left(\xi^{\prime}, \eta^{\prime}\right)=g\left(\xi^{\prime}-\eta^{\prime}, \tau\right)\left\langle\xi^{\prime}, \tau\right\rangle^{s}\left\langle\eta^{\prime}, \tau\right\rangle^{-s}$. Our goal is to show that $\int K\left(\xi^{\prime}, \eta^{\prime}\right) \mathcal{F}(v)\left(\eta^{\prime}, \tau\right) \mathrm{d} \eta^{\prime}$ is bounded in $L^{2}$. To do it, we will use Schur's Lemma. It suffices to prove that there exist $M>0$ and $N>0$ such that

$$
\int\left|K\left(\xi^{\prime}, \eta^{\prime}\right)\right| \mathrm{d} \xi^{\prime} \leq M \quad \text { and } \quad \int\left|K\left(\xi^{\prime}, \eta^{\prime}\right)\right| \mathrm{d} \eta^{\prime} \leq N
$$

In the sequel, we suppose $s \geq 0$ (the case where $s<0$ is treated in the same way).

For $R>0$, we have

$$
\begin{aligned}
\left\langle\xi^{\prime}, \tau\right\rangle^{2 R} g\left(\xi^{\prime}, \tau\right) & =\int\left\langle\xi^{\prime}, \tau\right\rangle^{2 R} \mathrm{e}^{-\mathrm{i} x^{\prime} \xi^{\prime}} \chi(x) \mathrm{e}^{\tau \varphi(x)} \mathrm{d} x^{\prime} \\
& =\int\left(1-\Delta+\tau^{2}\right)^{R}\left(\mathrm{e}^{-\mathrm{i} x^{\prime} \xi^{\prime}}\right) \chi(x) \mathrm{e}^{\tau \varphi(x)} \mathrm{d} x^{\prime} \\
& =\int \mathrm{e}^{-\mathrm{i} x^{\prime} \xi^{\prime}}\left(1-\Delta+\tau^{2}\right)^{R}\left(\chi(x) \mathrm{e}^{\tau \varphi(x)}\right) \mathrm{d} x^{\prime} .
\end{aligned}
$$

Then there exists $C>0$, such that

$$
\left|\left\langle\xi^{\prime}, \tau\right\rangle^{2 R} g\left(\xi^{\prime}, \tau\right)\right| \leq C \mathrm{e}^{C \tau}
$$

Moreover, we can write

$$
\int\left|K\left(\xi^{\prime}, \eta^{\prime}\right)\right| \mathrm{d} \xi^{\prime}=\int\left|g\left(\xi^{\prime}-\eta^{\prime}, \tau\right)\left\langle\xi^{\prime}-\eta^{\prime}, \tau\right\rangle^{2 R} \frac{\left\langle\xi^{\prime}, \tau\right\rangle^{s}\left\langle\eta^{\prime}, \tau\right\rangle^{-s}}{\left\langle\xi^{\prime}-\eta^{\prime}, \tau\right\rangle^{2 R}}\right| \mathrm{d} \xi^{\prime} .
$$

Using (4.67), we obtain

Since

$$
\int\left|K\left(\xi^{\prime}, \eta^{\prime}\right)\right| \mathrm{d} \xi^{\prime} \leq C \mathrm{e}^{C \tau} \int \frac{\left\langle\xi^{\prime}, \tau\right\rangle^{s}\left\langle\eta^{\prime}, \tau\right\rangle^{-s}}{\left\langle\xi^{\prime}-\eta^{\prime}, \tau\right\rangle^{2 R}} \mathrm{~d} \xi^{\prime}
$$

$$
\int \frac{\left\langle\xi^{\prime}, \tau\right\rangle^{s}\left\langle\eta^{\prime}, \tau\right\rangle^{-s}}{\left\langle\xi^{\prime}-\eta^{\prime}, \tau\right\rangle^{2 R}} \mathrm{~d} \xi^{\prime}=\int_{\left|\xi^{\prime}\right| \leq \frac{1}{\epsilon}\left|\eta^{\prime}\right|} \frac{\left\langle\xi^{\prime}, \tau\right\rangle^{s}\left\langle\eta^{\prime}, \tau\right\rangle^{-s}}{\left\langle\xi^{\prime}-\eta^{\prime}, \tau\right\rangle^{2 R}} \mathrm{~d} \xi^{\prime}+\int_{\left|\eta^{\prime}\right| \leq \epsilon\left|\xi^{\prime}\right|} \frac{\left\langle\xi^{\prime}, \tau\right\rangle^{s}\left\langle+\eta^{\prime}, \tau\right\rangle^{-s}}{\left\langle\xi^{\prime}-\eta^{\prime}, \tau\right\rangle^{2 R}} \mathrm{~d} \xi^{\prime}, \quad \epsilon>0 .
$$

If $\left|\xi^{\prime}\right| \leq \frac{1}{\epsilon}\left|\eta^{\prime}\right|$, we have

$$
\frac{\left\langle\xi^{\prime}, \tau\right\rangle^{s}\left\langle\eta^{\prime}, \tau\right\rangle^{-s}}{\left\langle\xi^{\prime}-\eta^{\prime}, \tau\right\rangle^{2 R}} \leq C \frac{\left\langle\eta^{\prime}, \tau\right\rangle^{s}\left\langle\eta^{\prime}, \tau\right\rangle^{-s}}{\left\langle\xi^{\prime}-\eta^{\prime}, \tau\right\rangle^{2 R}} \leq \frac{C}{\left\langle\xi^{\prime}-\eta^{\prime}, \tau\right\rangle^{2 R}} \quad \in L^{1} \quad \text { if } 2 R>n-1
$$

If $\left|\eta^{\prime}\right| \leq \epsilon\left|\xi^{\prime}\right|$, i.e. $\left\langle\xi^{\prime}-\eta^{\prime}, \tau\right\rangle \geq \delta\left\langle\xi^{\prime}, \tau\right\rangle, \delta>0$, we have

$$
\frac{\left\langle\xi^{\prime}, \tau\right\rangle^{s}\left\langle\eta^{\prime}, \tau\right\rangle^{-s}}{\left\langle\xi^{\prime}-\eta^{\prime}, \tau\right\rangle^{2 R}} \leq \frac{C}{\left\langle\xi^{\prime}-\eta^{\prime}, \tau\right\rangle^{2 R-s}} \quad \in L^{1} \quad \text { if } 2 R-s>n-1 .
$$

Then there exists $M>0$, such that

$$
\int\left|K\left(\xi^{\prime}, \eta^{\prime}\right)\right| \mathrm{d} \xi^{\prime} \leq M \mathrm{e}^{C \tau}
$$


By the same way, we show that there exists $N>0$, such that

$$
\int\left|K\left(\xi^{\prime}, \eta^{\prime}\right)\right| \mathrm{d} \eta^{\prime} \leq N \mathrm{e}^{C \tau}
$$

Using Schur's Lemma, we have $\left(\operatorname{op}\left(\Lambda^{s}\right) \mathrm{e}^{\tau \varphi} \chi \mathrm{op}\left(\Lambda^{-s}\right)\right)$ is bounded in $L^{2}$ and

$$
\left\|\operatorname{op}\left(\Lambda^{s}\right) \mathrm{e}^{\tau \varphi} \chi \mathrm{op}\left(\Lambda^{-s}\right)\right\|_{\mathcal{L}\left(L^{2}\right)} \leq C \mathrm{e}^{C \tau} .
$$

Applying in $\mathrm{op}\left(\Lambda^{s}\right) u$, we obtain the result.

\section{Appendix C: Proof of Theorem 1.1, For $\mu=0$}

Let $U=\left(u_{0}, v_{0}, v_{1}\right) \in D(\mathcal{A})$ and $F=\left(f_{0}, g_{0}, g_{1}\right) \in H$ such that $F=\mathcal{A} U$. Then we have the following system

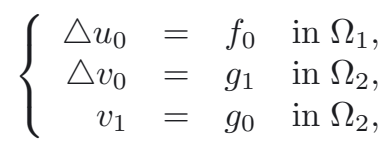

with the following boundary conditions

$$
\left\{\begin{aligned}
\left.u_{0}\right|_{\Gamma_{1}} & =0,\left.\quad v_{0}\right|_{\Gamma_{2}}=0 \\
\left.u_{0}\right|_{\gamma} & =\left.g_{0}\right|_{\gamma} \\
\left.\left(\partial_{n} u_{0}-\partial_{n} v_{0}\right)\right|_{\gamma} & =\left.0\right|_{\gamma} .
\end{aligned}\right.
$$

From $\Delta u_{0}=f_{0}$ in $\Omega_{1},\left.u_{0}\right|_{\Gamma_{1}}=0$ and $u_{0}=\left.g_{0}\right|_{\gamma}$, we have the following estimate

$$
\left\|u_{0}\right\|_{H^{1}\left(\Omega_{1}\right)}^{2} \leq C\left(\left\|f_{0}\right\|_{H^{-1}\left(\Omega_{1}\right)}^{2}+\left\|g_{0}\right\|_{H^{\frac{1}{2}}(\gamma)}^{2}\right) .
$$

Then

$$
\left\|u_{0}\right\|_{H^{1}\left(\Omega_{1}\right)}^{2} \leq C\left(\left\|f_{0}\right\|_{L^{2}\left(\Omega_{1}\right)}^{2}+\left\|g_{0}\right\|_{H^{1}\left(\Omega_{2}\right)}^{2}\right)
$$

Moreover, from $\triangle v_{0}=g_{1}$ in $\Omega_{2},\left.v_{0}\right|_{\Gamma_{2}}=0, \Gamma_{2} \neq \emptyset$ and $\partial_{n} v_{0}=\left.\partial_{n} u_{0}\right|_{\gamma}$, we have the following estimate

$$
\left\|v_{0}\right\|_{H^{1}\left(\Omega_{2}\right)}^{2} \leq C\left(\left\|g_{1}\right\|_{L^{2}\left(\Omega_{2}\right)}^{2}+\left\|\partial_{n} u_{0}\right\|_{H^{-\frac{1}{2}}(\gamma)}^{2}\right) .
$$

Recalling that $\triangle u_{0}=f_{0}$ and using the trace lemma (Lem. 3.4 in [6]), we obtain

$$
\left\|\partial_{n} u_{0}\right\|_{H^{-\frac{1}{2}(\gamma)}} \leq C\left(\left\|u_{0}\right\|_{H^{1}\left(\Omega_{1}\right)}+\left\|f_{0}\right\|_{L^{2}\left(\Omega_{1}\right)}\right)
$$

Combining with (4.69), we obtain

$$
\left\|v_{0}\right\|_{H^{1}\left(\Omega_{2}\right)}^{2} \leq C\left(\left\|g_{1}\right\|_{L^{2}\left(\Omega_{2}\right)}^{2}+\left\|u_{0}\right\|_{H^{1}\left(\Omega_{1}\right)}^{2}+\left\|f_{0}\right\|_{L^{2}\left(\Omega_{1}\right)}^{2}\right) .
$$

Combining with (4.68), we get

$$
\left\|v_{0}\right\|_{H^{1}\left(\Omega_{2}\right)}^{2} \leq C\left(\left\|f_{0}\right\|_{L^{2}\left(\Omega_{1}\right)}^{2}+\left\|g_{0}\right\|_{H^{1}\left(\Omega_{2}\right)}^{2}+\left\|g_{1}\right\|_{L^{2}\left(\Omega_{2}\right)}^{2}\right) .
$$

Recalling that $v_{1}=g_{0}$ and combining (4.68) and (4.70), we obtain Theorem 1.1, for $\mu=0$. 
Acknowledgements. Sincere thanks to professor Luc Robbiano for inspiring questions, his great contribution to this work and for careful reading of the manuscript. I want to thank also professor Mourad Bellassoued for his offer to work in this domain.

\section{REFERENCES}

[1] C. Bardos, G. Lebeau and J. Rauch, Sharp sufficient conditions for the observation, control, and stabilization of waves from the boundary. SIAM J. Control Optim. 30 (1992) 1024-1065.

[2] M. Bellassoued, Distribution of resonances and decay rate of the local energy for the elastic wave equation. Comm. Math. Phys. 215 (2000) 375-408.

[3] M. Bellassoued, Carleman estimates and distribution of resonances for the transparent obstacle and application to the stabilization. Asymptot. Anal. 35 (2003) 257-279.

[4] M. Bellassoued, Decay of solutions of the elastic wave equation with a localized dissipation. Ann. Fac. Sci. Toulouse Math. 12 (2003) 267-301.

[5] N. Burq, Décroissance de l'énergie locale de l'équation des ondes pour le problème extérieur et absence de résonance au voisinage du réel. Acta Math. 180 (1998) 1-29.

[6] T. Duyckaerts, Optimal decay rates of the energy of a hyperbolic-parabolic system coupled by an interface. Asymptot. Anal. 51 (2007) 17-45.

[7] X Fu, Logarithmic decay of hyperbolic equations with arbitrary small boundary damping. Commun. Partial Differ. Equ. 34 (2009) 957-975.

[8] J. Le Rousseau and L. Robbiano, Carleman estimate for elliptic operators with coefficients with jumps at an interface in arbitrary dimension and application to the null controllability of linear parabolic equations. Arch. Ration. Mech. Anal. (to appear).

[9] G. Lebeau, Équation des ondes amorties, in Algebraic and geometric methods in mathematical physics Kaciveli, 1993, Kluwer Acad. Publ., Dordrecht, Math. Phys. Stud. 19 (1996) 73-109.

[10] G. Lebeau and L. Robbiano, Contrôle exact de l'équation de la chaleur. Commun. Partial Differ. Equ. 20 (1995) 335-356.

[11] G. Lebeau and L. Robbiano, Stabilisation de l'équation des ondes par le bord. Duke Math. J. 86 (1997) 465-491.

[12] J. Rauch, X. Zhang and E. Zuazua, Polynomial decay for a hyperbolic-parabolic coupled system. J. Math. Pures Appl. 84 (2005) 407-470.

[13] L. Robbiano, Fonction de coût et contrôle des solutions des équations hyperboliques. Asymptot. Anal. 10 (1995) 95-115.

[14] M.E. Taylor, Reflection of singularities of solutions to systems of differential equations. Comm. Pure Appl. Math. 28 (1975) $457-478$.

[15] X. Zhang and E. Zuazua, Long-time behavior of a coupled heat-wave system arising in fluid-structure interaction. Arch. Ration. Mech. Anal. 184 (2007) 49-120. 